\title{
Thermohydrodynamics: Where Do We Stand?
}

\author{
L. S. García-Colín ${ }^{1,2}$, J. I. Jiménez-Aquino ${ }^{1}$ and F. J. Uribe ${ }^{1}$ \\ ${ }^{1}$ Universidad Autónoma Metropolitana - Iztapalapa \\ ${ }^{2}$ El Colegio Nacional. Centro Histórico, México D. F. \\ México
}

\section{Introduction}

The subjects selected to conform the content of this chapter have beem chosen following two main criteria. Firstly, subjects which are of great interest today in the field of thermohydrodynamics. Secondly, they are neither well understood nor we can regard them as fully developed. Many aspects in their own structure pose very deep and interesting questions whose answers are yet to be obtained. Indeed when closely examined one finds rather surprising results. Start with magnetohydrodynamics. When in a charged fluid ordinary hydrodynamics is coupled with Maxwell's equations using the conventional form of Ohm's equation an assumption lies behind which is hardly sustained with the results obtained in kinetic theory. Further, if the fluid is viscous such coupling becomes much more sophisticated a fact that it is hardly noticed in the current literature.

A similar situation occurs with shock waves when in spite of the fact Euler's equations are well known not to provide a correct description of the structure of a shock wave, they are still used in many applications. The use of the Navier-Stokes and higher order hydrodynamics equations seeking a more accurate description of shock waves is full of loopholes which we hope to bring to the attention of the reader.

On the other hand quantum hydrodynamics, a field long ignored by both physicists and hydrodynamicists is becoming more and more demanding specially due to the experimental results in relativistic heavy ion collisions (RHIC) and in the study of non-equilibrium properties of very cold Bose and Fermi gases. Indeed, one can safely assert that quantum hydrodynamics has never been correctly formulated.

The last topic we chose to discuss is currently referred to as Stochastic Thermodynamics and/or Fluctuation Theorems. This field pretends to extrapolate well established results in Thermostatics as well as in near-equilibrium thermodynamics in the study of small (nanoscale) systems. The two fundamental problems we see here is, firstly, the validity of the use of well defined macroscopic concepts to very small systems. This requires caution. Secondly, the abuse of the thermodynamic languaje in these systems is rather confusing. For instance, entropy is a state function only defined for equilibrium states of macrosystems or in near equilibrium states as required in linear non-equilibrium thermodynamics (LIT). Far away from equilibrium entropy is not defined and entropy production which is unfortunately adopted instead of Clausius uncompensated heat is only meningful in (LIT). In this part of the review we insist in placing the enormous amount of results so far obtained in a more adequate language. This will avoid misconceptions as well as misundertandings. 


\section{Magnetohydrodynamics}

In order to get a clear picture of what we are going to understand by magnetohydrodynamics, it is convenient to recall the methodology that lies behind the derivation of the equations of ordinary hidrodynamics. We consider a single fluid whose thermodynamic states are described by five locally conserved variables, $\rho(\mathbf{r}, t)$ the mass density, $\rho(\mathbf{r}, t) \mathbf{u}(\mathbf{r}, t)$ the momentum density, and $\rho(\mathbf{r}, t) e_{T}(\mathbf{r}, t)$ the total energy density. These equations satisfy the five well-known conservation equations, needless to be written down here. These equations contain fourteen unknowns, three for the energy flux, six for the momentum flux (assuming fluid is isotropic) and the five state variables. To turn them into a complete set of equations one needs to introduce nine so called constitutive equations, expressing the fluxes in terms of the state variables. For an ideal (non-viscous) fluid one readily obtains the well known Euler equations, the lowest order equations including dissipative effects are the Navier-Stokes-Fourier equations an so on.

If one now considers that the fluid is charged, then when set in motion its charge and current densities will generate an electromagnetic field which, through Maxwell's equations couples with the fluid's own hydrodynamic equations, giving rise to what is usually referred as the equations of magnetohydrodynamics. Yet the resulting equations are not closed. Constitutive equations must be supplied for all the resulting fluxes which arise from such coupling. Further, the state variables are now enlarged in number due to the presence of the charged particles. In the simplest case, say, the fluid consisting of electrons and ions, we must account for their corresponding densities. Needless to say that the resulting equations will be far more complicated that the ones for the ordinary fluid, a reason which, in our opinion, has given rise to some misunderstandings in the rich literature on the subject Alfven (1963); Balescu (1988); Braginskii (1965); Chandrasekhar (1960); Cowling (1957); García-Colín \& Dagdug (2009); Jackson (1962); Kulsrud (2005); Spitzer (1956); Van Kampen \& Felderhof (1967), including their kinetic theoretical foundations.

Consider a charged fluid consisting of electrons and ions of masses $m_{e}$ and $m_{i}$, respectively, whose number densities are $n_{i}$ and $n_{e}$ respectively. Then $\rho=n_{e} m_{e}+n_{i} m_{i}=\rho_{e}+\rho_{i}$ and the conservation equations read

$$
\begin{gathered}
\frac{\partial \rho}{\partial t}+\nabla \cdot(\rho \mathbf{u})=0 \\
\frac{\partial}{\partial t}(\rho \mathbf{u})+\nabla \cdot(\rho \mathbf{u} \mathbf{u}+\boldsymbol{\tau})=Q(\mathbf{E}+\mathbf{u} \times \mathbf{B})+\mathbf{J}_{c} \times \mathbf{B}, \\
\rho \frac{\partial \epsilon}{\partial t}+\nabla \cdot \mathbf{J}_{q}+\boldsymbol{\tau}:(\nabla \mathbf{u})^{s}+\mathbf{J}_{T} \times \mathbf{B}=0,
\end{gathered}
$$

where $\mathbf{u}(\mathbf{r}, t)$ is the fluid's velocity, $\boldsymbol{\tau}=p \mathbb{I}+\boldsymbol{\tau}^{v}, p$ being the hydrostatic pressure and $\boldsymbol{\tau}^{v}$ the symmetric viscous tensor, $\epsilon$ is the internal energy per unit mass, $\mathbf{J}_{q}$ the heat flux, $\mathbf{J}_{\mathcal{C}}$ the conduction current, and $\mathbf{J}_{T}$ the total current. Here $Q=n_{i} c_{i}+n_{e} c_{e}=\left(n_{i}-n_{e}\right) e$ and

$$
\mathbf{J}_{T}=Q \mathbf{u}+\mathbf{J}_{c}=Q \mathbf{u}+\frac{m_{e}+m_{i}}{m_{i} m_{e}} e \mathbf{J}_{e}^{(m)}
$$

where $\mathbf{J}_{e}^{(m)}$ is the mass current for the electrons. We recall that $\mathbf{J}_{e}+\mathbf{J}_{i}=0$. Maxwell's equations in the MKS system are,

$$
\nabla \cdot \mathbf{E}=\frac{1}{\epsilon_{0}} Q, \quad \nabla \cdot \mathbf{B}=0,
$$




$$
\nabla \times \mathbf{E}=-\frac{\partial \mathbf{B}}{\partial t}, \quad-\mu_{0} \epsilon_{0} \frac{\partial \mathbf{E}}{\partial t}+\nabla \times \mathbf{B}=\mu_{0} \mathbf{J}_{T}
$$

Notice that the currents $\mathbf{J}_{q}, \boldsymbol{\tau}^{v}, \mathbf{J}_{e}^{(m)}$ (or $\mathbf{J}_{c}$ ) require to be expressed in terms of the state variables $\rho_{e}, \rho_{i}, \mathbf{u}$, and $\epsilon$ through still unknown constitutive equations through eqs. (2) and (3).

In order to simplify the problem several approximations are introduced. Firstly the temperatures of electrons and ions is taken to be the same, $T_{i}=T_{e}=T$ a fact which is readily justified Moratto \& García-Colín (2011). Next we define the hydrodynamic time $\tau_{H}^{\alpha}$ and the hydrodynamic lenght $l_{H}$ as follows,

$$
\frac{1}{\tau_{H}^{\alpha}} \approx \frac{v_{\alpha}}{l_{H}}, \quad v_{\alpha}=\sqrt{\frac{2 k_{B} T}{m_{\alpha}}}, \quad \alpha=1, e,
$$

$k_{B}$ being Boltzmann's constant and

$$
l_{H}^{-1}=\operatorname{Max} \frac{|\nabla A(\mathbf{r}, t)|}{|A(\mathbf{r}, t)|},
$$

where $A$ is $\rho, \mathbf{u}$ or $T$.

We now assume that both $l_{H}$ and $\tau_{H}$ are the characteristic length and time scales of $\mathbf{E}$ and $\mathbf{B}$. Then several approximations follow Balescu (1988); García-Colín \& Dagdug (2009):

$$
\left|\frac{\partial \mathrm{E}}{\partial t}\right| \approx \frac{u^{2}}{c^{2}}|\nabla \times \mathbf{B}| \approx 0, \quad \text { in a non relativistic plasma } .
$$

This is a tricky approximation. By eq. (6) it implies that

$$
\nabla \times \mathbf{B}=\mu_{o} \mathbf{J}_{T}
$$

or that

$$
\nabla \cdot \mathbf{J}_{T}=0 .
$$

But since total charge is conserved, the balance equation

$$
\frac{\partial Q}{\partial t}+\nabla \cdot \mathbf{J}_{T}=0
$$

must be obeyed which implies that $\frac{\partial Q}{\partial t}=0$. Consistency may be achieved if local neutrality is satisfied namely Balescu (1988),

$$
\left|n_{e}(\mathbf{r}, t)-n_{i}(\mathbf{r}, t)\right| \leq \frac{1}{2}\left(n_{e}(\mathbf{r}, t)+n_{i}(\mathbf{r}, t)\right),
$$

in which case $Q \approx 0$ in all hydrodynamic equations.

This leads to the so called pre-Maxwell's equations $\left|\frac{\partial \mathrm{E}}{\partial t}\right| \approx 0$ and $Q \mathrm{E}$ is supressed. Moreover, it is also assumed that since $Q \approx 0$ then

$$
Q \mathbf{u}<<\mathbf{J}_{c}
$$

which implies that pre-Maxwell's equations read as

$$
\begin{aligned}
& \nabla \cdot \mathbf{E}=0, \\
& \nabla \times \mathbf{E}=-\frac{\partial \mathbf{B}}{\partial t}, \quad \nabla \times \mathbf{B}=\mu_{0} \mathbf{J}_{T},
\end{aligned}
$$


subject to the condition that $\nabla \cdot \mathbf{J}_{c}=0$.

This fact is very often ignored or by-passed in many treatises on the subject. If we assume that $\mathbf{J}_{c}$ is determined through Ohm's law, which as we will see below is already incorrect, then

$$
\mathbf{J}_{c}=Q\left(\mathbf{E}+\frac{1}{\mu_{0}} \mathbf{u} \times \mathbf{B}\right)=\frac{Q}{\mu_{0}}(\mathbf{u} \times \mathbf{B}),
$$

which implies that (eq. (16))

$$
-\nabla \times \mathbf{B}=Q(\mathbf{u} \times \mathbf{B})
$$

or

$$
\nabla \cdot(\mathbf{u} \times \mathbf{B})=0,
$$

which is consistent with eq. (11). But also,

$$
\nabla \cdot(\mathbf{u} \times \mathbf{B})=\mathbf{B} \cdot \nabla \times \mathbf{u}-\mathbf{u} \cdot \nabla \times \mathbf{B}
$$

or using eq. (18)

$$
\mathbf{B} \cdot \nabla \times \mathbf{u}=Q \mathbf{u} \cdot(\mathbf{u} \times \mathbf{B})=0 .
$$

This implies that, in general $\nabla \times \mathbf{u}=0$ or that the flow is always irrotational, which in turn implies the fluid is non-viscous. Whence, $\tau^{v}=0$ a fact that not always is clearly pointed out in many applications. Yet eq. (17) as such is completely incorrect. This is manifestly exhibited by kinetic theory Balescu (1988); Braginskii (1965); García-Colín \& Dagdug (2009).

To avoid unnecessary and lengthy repetitions we will explicitly consider the properties of vectorial currents, $\mathbf{J}_{i}^{(m)}$ and $\mathbf{J}_{q}$ but concentrate on the former one since identical properties are associated with the second one. Notice that from eq. (4) whatever we say about $\mathbf{J}_{e}^{(m)}$ applies to $\mathbf{J}_{c}$ since $m_{i}>>m_{e}$ and $\mathbf{J}_{c}=\left(e / m_{e}\right) \mathbf{J}_{e}^{(m)}$. Now, consider a fluid in which the magnetic vector $\mathbf{B}$ is along the $z$-axis, $\mathbf{B}=|B| \hat{\mathbf{k}}$. Any vectorial current has now three different components, one along the $z$-axis, one lying in a plane, the $x y$ plane perpendicular to $\mathbf{B}$ and a third one which lies on a plane perpendicular to both, the $z$-axis and the $x y$ plane. The two vectorial forces associated with vectorial fluxes are $\nabla T$ and $\mathbf{d}_{i e}$ where

$$
\mathbf{d}_{i e}=\nabla\left(\frac{n_{e}}{n}\right)+\frac{n_{i} n_{e}\left(m_{i}-m_{e}\right)}{n \rho} \frac{\nabla p}{p}+\frac{n_{i} n_{e}}{p \rho}\left(m_{i} e+m_{e} e\right) \mathbf{E}^{\prime},
$$

is the diffusive force and $\mathbf{E}^{\prime}=\mathbf{E}+\mathbf{u} \times \mathbf{B}$. If $n_{i}+n_{e}=\frac{n}{2}$ and we neglect the term with $\nabla p$ which is usually very small,

$$
\mathbf{d}_{i e}=\frac{n^{2}}{p \rho} m_{i} e \mathbf{E}^{\prime}
$$

ignoring also the temperature gradients which give rise to the Thomson effect systematically ignored in plasma physics. This implies that $\mathbf{J}_{C}$ may be written as (see refs. citemb8,mb10)

$$
\mathbf{J}_{c}=\sigma_{\|} E_{z} \hat{\mathbf{k}}+\sigma_{\perp}\left(E_{x} \hat{\mathbf{i}}+E_{y} \hat{\mathbf{j}}\right)+\sigma_{S}(\mathbf{E}+\mathbf{u} \times \mathbf{B}),
$$

where the three conductivities are explicitly given in terms of molecular parameters but we do not need them here explicitly. Equation (24) would be the canonical form of Ohm's law we repeat, ignoring pressure diffusion and Thomson's thermoelectric terms. Now compare eqs. (17) and (24). They would be identical if $\sigma_{\|}=\sigma_{\perp}=0$ and $\sigma_{S}=\sigma_{\|}$which is completely wrong. 
Even if we neglect $\mathbf{E}, \sigma_{S}$ is not by any chance the same as $\sigma_{\|}$as clearly exhibited in refs. Balescu (1988) and García-Colín \& Dagdug (2009).

A second critique arises from the use of pre-Maxwell's equations (15) and (16) subject to the condition that $\nabla \cdot \mathbf{J}_{c}=0 . \mathbf{J}_{c}$ is the outcome of the presence of all the thermodynamic forces present in the system $\nabla, \nabla p, \nabla \frac{n_{i}}{n}$, and $\mathbf{d}_{i e}$. To assert that in general $\nabla \cdot \mathbf{J}_{c}=0$ is ridiculous. Even if $\mathbf{B}=0$ one can show that García-Colín \& Dagdug (2009)

$$
\nabla \cdot \mathbf{J}_{c}=-\sigma_{\|} \nabla^{2} \phi-\tau_{\perp} \nabla^{2} T
$$

where $\tau_{\perp}$ is a thermal conductivity. Equation 25 set equal to zero, in general, cannot be satisfied unless $\nabla \phi$ and $\nabla T$ acted in a rather peculiar way.

As a third, and last critique we will mention here, concerns magnetohydrodynamics at the level of Euler's equations. Assuming $Q|\mathbf{E}|<<1$, Euler's equations read,

$$
\begin{gathered}
\frac{\partial \rho_{a}}{\partial t}=-\nabla \cdot\left(\rho_{a} \mathbf{u}\right), \quad a=i, e . \\
\rho \frac{\partial \mathbf{u}}{\partial t}=-\rho \mathbf{u} \cdot \nabla \mathbf{u}-\nabla p+\mathbf{J}_{c} \times \mathbf{B}, \\
\rho \frac{\partial T}{\partial t}=-\left(\mathbf{u} \cdot \nabla T+\frac{2 p}{3 n k_{B}} \nabla \cdot \mathbf{u}\right) .
\end{gathered}
$$

The full expression for $\mathbf{J}_{C}$ in the absence of viscosity is still required at this level! In this canonical form they are never used in the literature, except by setting $\mathbf{J}_{c}=1 / \mu_{0} \nabla \times \mathbf{B}$ but even if this expression is substituted in equation (27) one still needs the complete form for Jc to obtain B. Needless to say that if viscous effects are present the whole magnetohydrodynamic scheme becomes much more complicated and even for dilute gases, it appears that it has never been given its appropriate status. Just to mention one significative result, the stress tensor in the presence of a magnetic field is considerably modified giving rise to five viscosities, which are not always negligible when compared with the one that is not affected by the field, the standard shear viscosity of hydrodynamics. Details of these results are discussed in the literature Balescu (1988); Braginskii (1965); García-Colín \& Dagdug (2009) but they have been completely ignored. The literature on magnetohydrodynamics and plasma physics is overwhelming, in the partial list here provided more references can be found.

\section{Shock waves}

In the previous section some problems associated with magnetohydrodynamics (MHD) have been pointed out, so the reader may get the impression that for the case when there are no electromagnetic fields the situation is different, but there are cases in which the situation is far from being solved. Turbulence, sometimes referred as the last unsolved problem in classical physics, is an example in which the Navier-Stokes equations are used and their linearization destroys the problem Holmes et al. (1998). Therefore, turbulence is a genuine non linear problem but there are others like shock waves for which it is necessary to improve hydrodynamics beyond the Navier-Stokes description. For shock waves the Euler equations do not provide a strong solution (differentiable) and mathematicians have used the theory of weak solutions; these are discontinuous solutions or solutions in the sense of the theory of distributions developed by L. Schwartz Richtmyer (1978). In the Russian literature they are known as generalized functions and, as far we know, were introduced in physics by $\mathrm{P}$. 
Dirac (but perhaps the original idea goes back to Fourier). The famous Dirac's delta is an example of a "distribution" and it took a while to put it in a proper mathematical context. In the introduction of a book by von Neumann (1955) the reader can find a critique about distributions a la Dirac. From our point of view fluids are not ideal and the introduction of transport coefficients is necessary if one is interested in modeling nature properly. Therefore, we consider the Navier-Stokes equations as more appropriate than the Euler equations. In this sense the point of considering weak solutions is not important for us and we visualize this as a manifestation of the superiority of the physical content of the Navier-Stokes equations when compared to the Euler equations ${ }^{1}$. The reader may argue that at low temperatures $\mathrm{He}_{e}^{4}$ or $\mathrm{He}_{e}^{3}$ behave as fluids without viscosity (also Bose-Einstein condensates in dilute gases) so that the Euler equations should be relevant in this case. This is indeed a true but incomplete statement since according to Tisza and Landau the proper hydrodynamic description is in terms of two fluids: the normal fluid that has viscosity, and the superfluid that has no viscosity. Tieza-Landau's two fluid hydrodynamics is discussed in the next section on quantum hydrodynamics where the connection between quantum physics and classical hydrodynamics is also mentioned.

Apart from being a non linear problem, so that the whole set of equations of a theory are tested and not only its linearizations, shock waves are interesting in other aspects. For example, in astrophysics shock waves are found in many of the most spectacular events of nature Piran (2004); Woosley et al. (2002). They are generated in: explosions and detonations Fickett \& Davis (2000); Zel'dovich \& Raizer (2002), shock tubes Fomin (2010); Zel'dovich \& Raizer (2002), appear in: traffic flow Helbing (2001), sonoluminescence Brenner et al. (2002), inertial confinement Goncharov et al. (2006), and when vehicles enter the atmosphere of the earth or other planets Gnoffo (1999).

Classical hydrodynamics, or hydrodynamics for short in this section, was born as a continuum theory. The atomistic point of view of hydrodynamics, when available, can provide explicit expressions for the transport coefficients for an assumed interaction potential between the atoms (either analytically or numerically), so that one does not have to measure or guess them. Also, for simple systems like dilute gases the equation of state and caloric equation of state are known so that disagreement between theory and experiment (or simulations) point to inadequacies of the theory and not to incorrect transport coefficients or equations of state. This is the advantage of considering dilute gases where as a bonus the kinetic description provided by the Boltzmann equation is applicable (which, as far we know, physicists and mathematicians agree that it provides a sound description). The phenomenological derivation of the hydrodynamic equations is well documented in textbooks Currie (1974); Landau (1986), to mention just two among many others; the advantage of such derivation is that it applies to gases and liquids (fluids in general); its weakness (so to speak) is that the transport coefficients have to be determined from experiments, theory, or simulations. The most successful microscopic derivation of the Navier-Stokes equations that we know is that provided by using the Chapman-Enskog method Chapman \& Cowling (1970) to solve the Boltzmann equation

\footnotetext{
${ }^{1}$ It should be pointed out that being in a sense simpler than the Navier-Stokes, the Euler equations have perhaps a richer (perhaps simpler) mathematical structure and therefore quite a lot can be said from them, even for the MHD case. The book by V. I. Arnold is just an example Arnold (1998). On the other hand the one million dollar prize voiced by the Clay Mathematics Institute is for the Navier-Stokes equations only, but it is important to mention that Fefferman (2000) points out the relevance of Leray's existence theorem of weak solutions to the Navier-Stokes equations for tackling the problem of their existence and smooth solutions.
} 
2. Other books where one can find discussions about the Chapman-Enskog, Hilbert's method, and extensions for dilute polyatomic gases are: Cercignani (1988), Ferziger \& Kaper (1972), McCourt et al. (1990), Grad (1958), Résibois \& De Leener (1977), Sone (2002), and Struchtrup (2005), among others. The weakness (so to speak) of the microscopic approach is that its restriction to the very simple dilute gas leaves outside of its scope wealth of applications. In this section we will be concerned with some attempts to go beyond standard hydrodynamics using both the macroscopic and microscopic approaches.

In order to understand the aspects of the shock wave problem we consider the dilute gas where Boltzmann equation is available. For simplicity let us consider a plane shock wave so that the hydrodynamic variables are functions only of $x$, the position along the shock wave propagation, and time. The conservation equations for mass, momentum, and energy are the following Chapman \& Cowling (1970):

$$
\begin{gathered}
\frac{\partial}{\partial t} \rho(x, t)+\frac{\partial}{\partial x}(\rho(x, t) u(x, t))=0, \\
\rho(x, t)\left(\frac{\partial}{\partial t} u(x, t)+u(x, t) \frac{\partial}{\partial x} u(x, t)\right)=-\frac{\partial}{\partial x} P_{x x}(x, t), \\
\rho(x, t)\left(\frac{\partial}{\partial t} e(x, t)+u(x, t) \frac{\partial}{\partial x} e(x, t)\right)=-P_{x x}(x, t) \frac{\partial}{\partial x} u(x, t)-\frac{\partial}{\partial x} q(x, t),
\end{gathered}
$$

respectively, where the hydrodynamic velocity is given by $\mathbf{u}(\mathbf{r}, t)=u(x, t) \hat{\mathbf{i}}, \hat{\mathbf{i}}$ is a unit vector along the direction of propagation, $P_{x x}$ is the $x x$ component of the pressure tensor, and $q$ the heat flux. The previous conservation equations can be obtained from the Boltzmann equation or from the standard phenomenological approach Currie (1974) so that they seem to be well established. However, Brenner $(2005 ; 2006 ; 2010)$ has recently challenged them ${ }^{3}$; we will refer to his theory as the two velocity hydrodynamics. Except for this case all, the other theories that we will mention here rely on the standard conservations laws. The analysis done by Greenshields \& Reese (2007) on asymmetry factors show that in its present form Brenner's approach is not good for explaining the shock wave problem. Another phenomenological approach is that by Hoover \& Hoover (2010b) who propose a two temperature theory with delays to try to account for new results obtained using the smooth particle method for shock waves Hoover \& Hoover (2010a), further analysis of this theory Uribe et al. (2011) reveal the existence of singularities but it is necessary to analyze it with more detail. Meanwhile Hoovers' ideas have been modified to produce the so-called Burnett-Cattaneo continuum theory for shock waves Holian et al. (2011).

The shock wave problem consists in solving the conservation equations (29)-(31), either for the non-stationary or stationary case, given the Rankine-Hugoniot jump conditions that

\footnotetext{
${ }^{2}$ There is no universal agreement on this statement; Truesdell \& Müncaster (1980) consider the Chapman-Enskog method, as given by Chapman \& Cowling Chapman \& Cowling (1970), as "naive" (to say the least) and proposed the methods of stretched fields and the Maxwellian iteration to solve the kinetic problem. The mathematical aspects of the Boltzmann equation and in particular the results by DiPerna \& Lions can be found in the literature Cercignani et al. (1994); Villani (2002).

${ }^{3}$ The modification to the conservation equations by Brenner can briefly be explained as follows: conservation of mass and momentum remain the same but in eq. (31) the heat flux q should be changed by the diffusive volume flux density $\left(\mathbf{j}_{e}=\mathbf{u}_{m}-\mathbf{u}_{v}\right.$ ), where $\mathbf{u}_{m}$ is the velocity that appears in the conservation equations and $\mathbf{u}_{v}$ is the velocity that appears in the constitutive equations. This idea of two velocities is unusual and the reader is referred to Brenner's works for more explanations. We recommend the paper by Greenshields \& Reese (2007) for additional explanations of Brenner's ideas, they analyzed Brenner's theory for the shock wave problem.
} 
are obtained from the steady form of the conservation equations. These two conditions of pressure, velocity, etc. are identified with equilibrium thermodynamic states and the goal is to see how the equilibrium points are connected as a function of $x$, say. As mentioned before the Euler equations do not provide a connection between the two equilibrium points but the Navier-Stokes do. Unfortunately the way in which they are connected is not accurate when compared with experimental information Alsmeyer (1976) or simulations like the Direct Simulation Monte Carlo Method (DSMC) Bird (1994) or Molecular Dynamics (MD) Salomons \& Mareschal (1992) for dilute gases ${ }^{4}$.

In the three conservation equations (29)-(31) we have the five unknowns $\left\{\rho, u, e(\right.$ or $\left.T), P_{x x}, q\right\}$ and additional equations are required. There are two main theoretical pathways that can be classified according to the way in which the additional information is provided:

- "Normal" solutions: the goal is to express the fluxes, $P_{x x}$ and $q$, in terms of the conserved variable $\{\rho, u, e\}$ and their gradients. Under this category we find the Navier-Stokes equations, the Burnett equations, super-Burnett equations, and so on Burnett (1935); Chapman \& Cowling (1970); Foch (1973); Foch \& Simon (1977); Uribe et al. (2000; 1998). They can be obtained using the Chapman-Enskog theory to solve the Boltzmann equation and in the particular case of the Burnett equations they can be obtained also using the methods by Truesdell and Müncaster Truesdell \& Müncaster (1980) (the method of stretched field and the Maxwellian iteration). Sometimes the form of the collision operator is changed, the most common model being that by Bhatnagar, Gross, and Krook (BGK) Bhatnagar et al. (1954), from which one may generate what are called BGK-Burnett equations an so on.

Due to the problems associated with the Burnett equations a la Chapman-Enskog, modifications to them have been proposed. They may be classified according to;

Subsets or supersets of the Burnett equations Fiscko \& Chapman (1989)

Regularizations Jin \& Slemrod (2001); Rosenau (1989); Slemrod (1998)

Generalizations Bobylev $(2008 ; 2011 ; 2006)$

BGK-Burnett Agarwal et al. (2001); Xu \& Li (2004)

It is interesting to notice that the Burnett equations have been used recently in the field of granular material Brey et al. (1998); Jin \& Slemrod (2001); Sela \& Goldhirsch (1998). A review of Bunett hydrodynamics is available for the interested reader García-Colín et al. (2008), also available is the detailed work by Agarwal et al. (2001) where the reader can find futher descriptions of such equations and their variants.

- Moment methods: Here additional equations (usually of the relaxation type) for $\left\{P_{x x}, q\right\}$ are provided. The main idea is to consider an expansion of the distribution function in terms of a complete set (usually Hermite tensors as done by Grad (1958)) in the velocity space whose coefficients are the so called moments that are functions of position and time. Hence one must truncate the expansion (closure) of the distribution function to get a finite number terms. Having done this one obtains the equations for the moments by using the Boltzmann equation. The well-known Grad's 13-moment approximation provides the "minimal" approximation that contains as moments the viscous pressure tensor and

\footnotetext{
${ }^{4}$ There is an enormous literature on MD in dense gases, liquids, and solids but we refer to just two works Holian \& Lomdahl (1998); Holian et al. (1980). Another powerful computational tool is the Smooth Particles Method (SPM) Hoover (2006), originally developed for astrophysical problems, which has been applied for the shock wave problem Hoover \& Hoover (2010a).
} 
the heat flux, so that the equations for the moments provide the additional equations to close the conservation equations for the shock wave problem. Several closures have been considered in the literature, for instance:

Grad's 13-moment approximation Grad (1958).

Regularized 13-moment approximation (R13) Struchtrup (2005).

Eu's theory Eu (1998).

Müller \& Ruggeri (1993).

There are also relations between normal solutions and moments methods, in particular between the Burnett equations and Grad's 13-moment equations using the Maxwell-Truesdell-Green iteration Agarwal et al. (2001) (called Maxwellian iteration by Truesdell \& Müncaster (1980)). One can also perform a Knudsen expansion of Grad's 13-moment approximation to obtain the Navier-Stokes equations.

In our opinion a main objection to moment methods concerns the problem related to the aforementioned truncation ${ }^{5}$. For certain cases it has been necessary to use hundreds or thousands of moments Müller \& Ruggeri (1993) to get agreement with experimental data, this approach therefore lacks predictive power in the sense that while agreement with experiment can be obtained, one is never sure what should be the number of moments to consider in a situation where there is no experimental information. Nevertheless one may consider more and more moments till the results do not change anymore (this is done in computational physics). On the other hand, the goal of having only a few moments to describe the systems like the Navier-Stokes equations is lost.

In addition to the two general methods mentioned and their variants, there are at least three other approaches that have been used for shock waves. They are: the Mott-Smith anzats Mott-Smith (1951), the Holian's conjecture Holian et al. (1993), and multi-temperature methods Xu \& Josyula (2005). For a critique of the former approach see García-Colín et al. (2004).

All this discussion raises an important question namely, where do we stand on the description for shock waves for a dilute monatomic gas? For the Navier-Stokes equations the situation is well understood since the works by Gilbarg (1951) and Gilbarg \& Paolucci (1953). With respect to the agreement with the experiment it should be pointed out that the comparisons become more sophisticated. There are several levels of comparisons that can be performed; shock thickness ${ }^{6}\left(\left(u_{0}-u_{1}\right) /|d u / d x|_{\max }\right)$, profiles $(u, T$, or $\rho$ vs. $x)$, orbits (or their projections) of the underlying dynamical system, and the asymmetry factor $\left(Q_{F}\right)^{7}$. It became clear that the Navier-Stokes equations were not good enough to provide a faithful representation of the information gathered from experiments and simulations. The consideration of the asymmetry factor provides compelling evidence of this Alsmeyer (1976) and actually in our opinion this quantity should always be evaluated. That the consideration of the profiles is not enough can be exemplified with Holian's conjecture Holian et al. (1993). This conjecture leads to better profiles but when the orbit is considered it yields the same orbit as the Navier-Stokes equations. This was noted computationally Uribe et al. (2000) and later on analytically Uribe (2001), so one is lead to the conclusion that the resulting dynamical system

\footnotetext{
${ }^{5}$ For other critique to moment methods see García-Colín et al. (2004); Velasco et al. (2002).

${ }^{6} u_{0}$ and $u_{1}$ refer to the equilibrium values of the velocity given by the Rankine-Hugoniot conditions.

${ }^{7}$ Here we are interested in the hydrodynamic description and so we prefer not to mention the comparisons at the level of the distribution function that are available Erwin et al. (1991), another quantity of interest is the temperature-density separation Greenshields \& Reese (2007).
} 
obtained from the conjecture is just a way to parametrize the Navier-Stokes equations. Recently, it has been shown that in appropriately reduced variables the orbits resulting from the Navier-Stokes equations are the same independently of the interaction potential when one relates the viscosity and thermal conductivity according to the prediction resulting from the first Sonine expansion when the Chapman-Enskog method is considered Uribe (2011). With respect to the theories for which an evaluation of the asymmetry exists we mention the following; Navier-Stokes Alsmeyer (1976); Pham-Van-Diep et al. (1991), Brenner's Greenshields \& Reese (2007), R13 Struchtrup (2005), Burnett Pham-Van-Diep et al. (1991); Uribe et al. (2000), and Fiscko \& Chapman (1989). The predictions of the last three (R13, Burnett, and Fiscko \& Chapman) are similar with respect to the asymmetry factor and are consistent with simulations and experimental data. However, except for the one by Fiscko \& Chapman (1989), the other two lack solutions ${ }^{8}$ for Mach numbers greater than about $5 / 2$. Brenner's two velocity hydrodynamics predicts asymmetry factors that are too low for Mach numbers greater than about 3 and its trend is different from the experimental one. The Navier-Stokes equations provide values for the asymmetry factors that are too big for Mach numbers form one up to two.

While we have not been exhaustive with the many approaches in the literature we think that the reader can get an idea of the complexity of the field of extending the Navier-Stokes equations. In our opinion the large number of approaches available is just a manifestation that there is no theory in the field with which researchers agree. This has lead to fragmentation and no synthesis seems possible in the near future. It would be very convenient to have asymmetry factors for all the other theories mentioned since then we could have a way of discriminating among the vast number available. In our opinion the procedure of adding or eliminating terms from hydrodynamic theories to eliminate problems of a theory or to get agreement with the experimental data should not be encouraged since predictive power most likely will be lost Fiscko \& Chapman (1989).

\section{Quantum hydrodynamics}

In 1927 the German physicist E. Madelung (1927) published a paper entitled Quantum Theory in hydrodynamical form. There he showed how Schrödinger's equation for electron systems could be cast into the form of the mass and momentum conservation equations for the system. After some pertinent comments on their properties he discussed possible applications without attempting any further efforts to derive transport equations. Curiously enough, in 1941 in his landmark paper on the theory of superfluidity Landau (1941), Landau devoted the first section of his paper to derive from quantum mechanical arguments Euler's equation describing the motion of inviscid but otherwise arbitrary fluid. These equations were written in operational form and he never referred to Madelung's work. More surprisingly, he never went beyond this stage but used general features of what he considered some basic aspects of quantum hydrodynamics to obtain the qualitative form of the energy spectrum of a quantum liquid, applying the results to $\mathrm{He}_{e}^{4}$ and from the well known form London (1964); Wilks (1966); Yarnell et al. (1959) of such spectrum computed the temperature dependance of thermodynamic functions for this system. And further, without invoking any other quantum mechanical aspects of liquid helium he set forth, using only the form of such spectrum, his well known two-fluid theory of superfluid helium $\mathrm{He}_{e}^{4}$. The equations of the macroscopic hydrodynamic of this fluid are all expressed in classical language, so the question arises about

\footnotetext{
${ }^{8}$ It is not known if solutions for Mach numbers greater than about $5 / 2$ exist.
} 
how, being a quantum fluid, there is no obvious presence of Planck's constant $\hbar$. This is not so and the corresponding situation has been clearly and exhaustively discussed in the literature Khalatnikov (1965); London (1964); Putterman (1974). Just to emphasize the point, the equation of state for the superfluid density $\rho_{S}$ as a function of $T$, the temperature, $p$ the pressure and $\left(\mathbf{v}_{n}-\mathbf{v}_{s}\right)^{2}$ the square of the difference between the velocities of the normal fluid $\mathbf{v}_{n}$ and the superfluid $\mathbf{v}_{S}$, contain $\hbar$. However, this is not a hydrodynamic affair. In fact, the quantum macroscopic aspects of the theory appeared explicitly when Onsager (1948) and Feynman (1955) independently proposed that in order to account for vorticity in the superfluid, the circulation in it should be quantized, namely,

$$
\oint \mathbf{v}_{S} \cdot d \mathbf{l}=n \frac{h}{m}
$$

where $\mathrm{n}$ is an integer. Besides the many important results obtained from this proposal Khalatnikov (1965); Putterman (1974), the two fluid theory plus the quantization of vorticity may be regarded as a quantum hydrodynamical theory. Nevertheless, this is not entirely satisfactory. $H_{e}^{4}$ is a many body boson system interacting among themselves with well known potentials and therefore one could ask the question as to how can one derive the two fluids hydrodynamic equations starting from the basic principles of quantum mechanics. The answer is, up to date, a completely open one. Not even the existence of a pure quantum Boltzmann equation from which we could extract, in analogy to the classical case, the hydrodynamics of a dilute Bose gas is available. Efforts have been made in this direction but they are still incomplete. Some progress was made by K. Huang (1964) for a hard sphere Bose gas but, once more, heuristic assumptions could not be avoided.

Another route followed by some authors was based on the well known Wigner's distribution functions. Proposed in 1932, this author Wigner (1932) defined quantum mechanical distributions in phase space, an apparent contradiction with the uncertainty principle, but more as a tool to perform practical calculations than to solve the deep nature of quantum statistical mechanics. Indeed, in spite of exhibiting the possibility of generating negative values they gave rise to a scheme which in principle allowed the calculation of ensemble averages properly defined of microscopic quantities which in turn provided their macroscopic counterparts Irving \& Zwanzig (1951); Mori et al. (1962). However, very few applications of practical utility were ever done although, curiously enough, for non-equilibrium dilute systems a Boltzmann like equation could be derived Mori et al. (1962) from which Euler's equations for a non-viscous fluid followed at once Irving \& Kirkwood (1950). Applications to the well known correlation functions method for linear dissipative systems were also made Mori et al. (1962) but once more, the ensuing calculations never went beyond low density gases.

The reader may immediately wonder about the status of this problem in the case of Bose condensates. This system, which has been rather fashionable and subject to a great deal of attention for the past two decades, poses even more complicated features. The atoms in which it has been studied are mostly composed of alkali atoms so their potential energy is far more sophisticated than in $\mathrm{He}_{e}^{4}$ so it is very doubtful if any systematic studies have been carried on their hydrodynamic properties. A similar comment follows for cold Fermi gases. Indeed, what one should expect is that from the N-body Schrödinger equation, using the appropriate symmetry requirements for the corresponding wave functions for fermions and bosons would lead to the conservation equation for particle, momentum and energy densities. 
These equations supplied with constitutive equations, either drawn from the experiment or derived from kinetic type equations, would finally yield the transport equations together with meaningful expressions for the transport coefficients. Although an attempt has been made in the past using the quantum analogy of the classical BBGKY hierarchy for reduced density matrices progess was strongly hindered, as in the classical case, due to difficulties related to truncation criteria and explicit solutions for the equations containing the remaining density matrices. A long and rather suggestive approach following these ideas was discussed almost thirty years ago Kadanoff \& Baym (1962); Putterman (1974). The interested reader should take a look of this work. In the absence of a program of this sort what we could consider the quantum mechanical approaches to the study of transport properyies of bosons and fermions are of a wide variety. A very thorough and exhaustive review of these methods has been published recently Schäfer \& Teaney (2009) and the interested reader is strongly encouraged to look at it. Briefly speaking the starting point is the concept of a "good fluid" namely, a fluid characterized by a small shear viscosity. To characterize these type of fluids one takes the expression for the shear viscosity for a simple gas as derived from elementary kinetic theory Kauzmann (1966); Reif (1966)

$$
\eta=\frac{1}{3} n p l
$$

where $n$ is the density, $p$ the average momentum of the particles and $l$ the mean free path. In the strong coupling limit, $l$ is very small so that the uncertainty relation implies that

$$
p l \sim \hbar
$$

so from eqs. (33) and (34) we infer that $\eta \sim n \hbar / 3$. Now, we know that for many fluids, bosons and fermions in the non relativistic limit, $s / n \sim k_{B}$ where $s$ is the entropy density so, for good fluids we may estimate that

$$
\frac{\eta}{S} \sim \frac{\hbar}{k_{B}}
$$

where $k_{B}$ is Boltzmann's constant. This result has even lead to the conjecture that, using results obtained from string theory Kovtun (2005)

$$
\frac{\eta}{s} \geq \frac{\hbar}{4 \pi k_{B}}
$$

¡for all fluids! When the equality holds as it appears to be the case in the quark-gluon plasma obtained in relativistic heavy ion collisions (RHIC) Aad et al. (2010); Aamodt (2010) the fluid is defined as a "perfect" one. Wether eq.(36) is completely correct or not is yet an unsolved problem but most of the advances that have been reported in the literature Schäfer \& Teaney (2009) are strongly bound to this question.

Just as an illustration of the ideas which are in close and deep scrutiny today, eq. (36) has been both examined experimentally and theoretically in a unitary Fermi Gas Cao et al. (2011). This gas is one in which by the use of a magnetic field the range of the interacting potential is not to be small when compared with the inter-particle spacing. Under these circumstances the properties of the gas turn out to be universal functions of the density $n$ and the temperature $T$. For the equilibrium properties the details have been recently revised Horikoshi (2010); Luo (2009). For the non-equilibrium properties the studies mentioned above Schäfer \& Teaney 
(2009) also hint at the existence of a universal hydrodynamics which appears to be consistent with eq. (36). If the findings turn out to be also applicable to both cold Bose and Fermi liquids we shall be contemplating a formidable breakthrough in quantum hydrodynamics.

Nevertheless there are still other efforts that have been advanced in connection with the problem of quantum hydrodynamics. These are based on the pioneer work on the subject by Landau who in 1953 Landau (1953) developed a description for the evolution of dense matter at high temperatures and pressure. This description was later improved by Hwa (1974) and Bjorken (1983) and very recently, re-examined thoroughly by C. Y. Wong (2008) specifically focused to account for the experimental data that is available from RHIC. The recent results obtained by this author are also quite interesting specially because the method itself is quite independent of the methods described in the previous paragraphs and quite free from the more complicated techniques of quantum field theory.

To finish this section we must briefly mention an attempt to analyze the transport problems of dense nuclear matter at very high temperatures under conditions that not only quantum mechanical equations are required, but also their relativistic versions. The ensuing formalism may be loosely regarded as the relativistic generalization of Madelung's early attempt as described at the beginning of this section. The method itself, which has a long history Wong (2010), curiosly enough, started from the Klein-Gordon equation in spite of the fact that, as well known, has a probability density $\rho=2 \operatorname{Im}\left(\Psi^{\star} \frac{\partial \Psi}{\partial t}\right)$ which is not necessarily a positive quantity. Nevertheless, Feshbach \& Villars (1958) were able to overcome this inconvenience by noting that the Klein-Gordon equation constrains both, particle and antiparticle degrees of freedom. That motivated Wong to write such equation in hydrodynamic form first for a single particle and then for N-body systems with strong interactions Wong (2010). Although one can indeed obtain rather complicated equations in which there are terms which may be identified, for instance, with a quantum stress tensor the whole scheme is still too formal to allow explicit calculations that may be related with transport properties, even less with transport coefficients. The reader interested in more details about this formalism should go to the original sources, it is beyond this review to provide the details which, one must say, are rather elaborated.

Summing up, this brief review clearly reveals that a quantum hydrodynamical theory is far from being developed. Even at the level of dilute systems the quantum analogue of Boltzman's equation is not available. The study of transport phenomena in quantum systems has thus followed a variety of routes which, in spite of their success, still offer no hint as to how to solve the general problem.

\section{Fluctuation theorems and stochastic thermodynamics}

It is well known that equilibrium statistical mechanics is a well established theory because it can explain the properties of a broad variety of systems in equilibrium. Close to equilibrium the linear response formalism is very successful in the form of the fluctuation-dissipation theorem and Onsager's reciprocity relations. However, no such universal framework exists to study systems driven far away from equilibrium. Over the past two decades, there has been considerable interest in the study of nonequilibrium statistical mechanics of small systems in which thermal fluctuations play a very important role, and has led to the discovery of several rigorous theorems called fluctuation theorems (FTs) and related results for systems away from 
equilibrium ${ }^{9}$. The fluctuation theorem (FT) deals with the relative probability that a quantity so-called "entropy" of a system which is currently away from thermodynamic equilibrium will increase or decrease over a finite time interval. The second law of thermodynamics states that the Clausius entropy of a macroscopic isolated system always increases until reaches equilibrium. The entropy and the entropy production as well, are quantities associated to macroscopic systems and they have a clear physical meaning as properties of ensembles. With the discovery of statistical mechanics it was shown that the entropy increase principle is only statistical one, (it is valid as an average over the ensemble) suggesting that there always exists some nonzero probability that the entropy of a isolated system might spontaneously decrease. The fluctuation theorem precisely quantifies this probability. So, when the thermodynamic concepts are applied to small systems and in short time intervals, it becomes clear that there exists a probability to find situations which do not match the ensemble averages. The FT was first proposed and tested using computer simulations, by Evans et.al Evans et al. (1993) in 1993. The first mathematical proof was given by Evans and Searles Evans \& Searles (1994) in 1994. Roughly speaking, the FT is related to the probability distribution of the time-average irreversible "entropy like production", denoted as $\bar{\Sigma}_{t}$. The theorem states that, in systems away from equilibrium over a finite time $t$, the ratio between the probability that $\bar{\Sigma}_{t}$ takes a value $A$ and the probability that it takes the value $-A$, will be exponential in At. In other words, for a finite non-equilibrium system in a finite time, the FT gives a precise mathematical expression for the probability that such a quantity will flow in an opposite direction. Its mathematical expression reads $P\left(\bar{\Sigma}_{t}=A\right) / P\left(\bar{\Sigma}_{t}=-A\right)=e^{A t}$. Because to $\bar{\Sigma}_{t}$ is an extensive quantity, it can be seen from the expression that, as the time or system size increses, the probability of observing the value $-A$ in opposite direction decreases exponentially, consistently which one could expect with the increase in entropy principle. Notice that the FT does not state that the second law of thermodynamics is wrong or invalid. Thus the FT when applied to macroscopic systems, is equivalent to the increase in entropy principle. Actually, the FTs involve a wide class of systems as well as several equilibrium and nonequilibrium quantities, including Helmholtz free energy Crooks (2000; 1999); Jarzynski (1997a;b; 2007); Jayannavar \& Sahoo (2007); Jiménez-Aquino et al. (2008), work Jayannavar \& Sahoo (2007); Jiménez-Aquino et al. (2008); Roy \& Kumar (2008); van Zon \& Cohen (2003), heat van Zon \& Cohen (2004), and entropy production Crooks (2000; 1999); Jiménez-Aquino (2010); Saha et al. (2009); Seifert (2005; 2008). They can be applied to steady state situations Gallavotti \& Cohen (2005); Jiménez-Aquino et al. (2010); van Zon \& Cohen (2003) and the transient theorems allow to go a step further Crooks (1999); Evans et al. (1993); Jiménez-Aquino et al. (2010); Jiménez-Aquino (2010); Saha et al. (2009); Seifert (2005); van Zon \& Cohen (2003). The FTs have been corroborated by both computer simulations Evans et al. (1993); Lechner et al. (2006); Searles \& Evans (2000); Wang et al. (2002)

\footnotetext{
${ }^{9}$ A short list of relevant works is the following; Evans et al. (1993), Evans \& Searles (1994), Evans \& Searles (2002), Jarzynski (1997a), Jarzynski (1997b), Jarzynski (2007), Liphardt et al. (2002), Esposito \& Van den Broek (2010), Shargel (2010), Sevick et al. (2008), Crooks (1999), Crooks (2000), Collin et.al. (2005), Garnier \& Ciliberto (2005), van Zon \& Cohen (2003), van Zon \& Cohen (2004), Seifert (2005), Seifert (2008), Saha et al. (2009), Gallavotti \& Cohen (2005), Searles \& Evans (2000), Lechner et al. (2006), Wang et al. (2002), Carberry et al. (2004), Trepagnier et al. (2004), Blickle et al. (2006), Tietz et al. (2006), Esposito et al. (2010), Saito \& Dhar (2007), Campisi et al. (2009), Ritort (2009), Velasco et al. (2011), Jayannavar \& Sahoo (2007), Saha \& Jayannavar (2008), Roy \& Kumar (2008), Jiménez-Aquino et al. (2008), Jiménez-Aquino et al. (2009), Jiménez-Aquino et al. (2010), Jiménez-Aquino (2010), Bustamante et al. (2005), Zuckerman \& Wolf (2002), Procacci et al. (2006), Sekimoto (1998), Carrete et al. (2008).
} 
and experiments Blickle et al. (2006); Carberry et al. (2004); Tietz et al. (2006); Trepagnier et al. (2004); Wang et al. (2002). The FTs are not only active areas of research in classical physics but recently also in quantum mechanics Campisi et al. (2009); Esposito et al. (2010); Saito \& Dhar (2007). The first laboratory experiment performed to demonstrate the transient work-fluctuation theorem (TFT) was reported by Wang et al. Wang et al. (2002) in 2002, for a small system over short times. In this experiment the trajectory of a colloidal particle is followed when it is captured in an optical trap that is translated relative to surrounding water molecules (the bath at temperature T).

After the works of Evans, Searles, and colleagues, another important result for non-equilibrium properties, called the Jarzynski equality (JE) Jarzynski (1997a;b; 2007) appeared in 1997 . This equality relates averages of non-equilibrium work distribution with the equilibrium free energy differences between equilibrium states. In other words, a system in thermodynamic equilibrium with a thermal bath at temperature $T$ can be driven out of equilibrium by time-dependent external forces. For slow enough driven forces, the process is quasistatic or reversible, then the system evolves through a succession of equilibrium states and the total amount of work $W_{\text {rev }}$ done by the external forces is equal to the equilibrium free-energy difference $\Delta G$ between the initial and final state. For nonequilibrium situations (irreversible processes), the second law of thermodynamics tell us that the total work $W$ is larger than the reversible work: the difference between the actual work and reversible work is equal to the net heat (noncompensated Clausius' heat) produced by dissipative forces and transferred from the system to the heat bath. Jarzynski found for arbitrary irreversible processes the relation $\left\langle\exp \left(-W / k_{B} T\right)\right\rangle=\exp \left(-\Delta G / k_{B} T\right)$, where $\langle\ldots\rangle$ denotes the average over an infinite number of trajectories or realizations of the irreversible process, $k_{B}$ is the Botzmann constant. The reversible process is then a particular case of this realation where $W=\Delta G$. The first experimental test of the JE was performed by Bustamante and collegues Liphardt et al. (2002) at UC Berkeley in 2002. They measured the work exerted on small RNA molecules using optical tweezers. In 1999, G. Crooks Crooks (1999) also at UC Berkeley, was able to go further by generalizing the JE. The Crooks relation states that the for a given protocol, the ratio between the distribution of work $P(W)$ measured along a forward trajectory (or probabilty distribution along a forward process) and its time reverse counterpart $P(-W)$ is given by $P(W) / P(-W)=\exp \left[(W-\Delta G) / k_{B} T\right]$. In 2005, this flutuation relation was experimentally tested by C. Collin el al. Collin et.al. (2005), using RNA molecules. Coming back to the experimental demonstration by Wang et al. Wang et al. (2002), they succeeded to test the TFT for a colloidal particle trapped into an optical trap which interacts with an experimental vessel, and the expression for the TFT reads $P(W) / P(-W)=e^{W}$, where $W$ stands for the dimensionless work delivered to the vessel, $P(W)$ is the probability of the work delivered to the vessel (or the probabilty of the work measured along a forward trajectory), and $\mathrm{P}(-\mathrm{W})$ is the probability that the vessel does work on the trap (or the probabilty of the work measured along a backward trajectory). After Wang's et al., Liphardt et al. and Collin et al. experimental confirmations, others were continued with colloidal particles trapped by an optical device, for instance Blickle et al. (2006); Carberry et al. (2004); Trepagnier et al. (2004), mechanical and electrical oscillators Garnier \& Ciliberto (2005), etc. For all of those statements given above, it becomes clear that the FTs are very important in the description of non-equlibrium systems within short times, and suggest that the miniaturization of machines may have inherent limitations. This means that microscopic (nanosized) machines will work not in the same way as their macroscopic counter parts. As the machines become smaller, the probability that they run in reverse 
becomes greater. In general, whatever is the non-equilibrium quantity, work, heat, entropy production, etc., the FTs relate probability distribution functions along forward and backward trajectories for a small system. The specific form of the various theorems depend on which thermodynamic parameter (temperature, volume, etc.) are held constant, wheter the system is prepared in an equilibrium state, and other factors.

The FTs have also been verified for heat van Zon \& Cohen (2004) and entropy like production Saha et al. (2009); Seifert $(2005 ; 2008)$. In the particular case of the entropy production, it has been necessary to define an entropy-like function along a stochastic trajectory. This and other thermodynamic concepts like the first-law-like energy balance involving applied or extracted work, exchanged heat, and changes in internal energy, were consistently defined along a single stochastic trajectory in the context of stochastic thermodynamics by Seifert Seifert (2005; 2008). Stochastic thermodynamics has been developed for mesoscopic systems like colloidal particles or single (bio) molecules driven out of equilibrium by time-dependent forces but still in contact with a heat bath of well-defined temperature. Stochastic thermodynamics thus attempts to extrapolate thermodynamic concepts to single particle trajectories. But one can immediately ask, how this extrapolation can be done? First of all, it is well known from the equilibrium statistical mechanics that the thermodynamics quantities such as the internal energy, entropy, etc., are related to ensemble properties and the average over the ensemble for each one becomes its thermodynamic counterpart. A similar situation has been adopted in stochastic thermodynamics by Seifert Seifert $(2005 ; 2008)$, in which that concept of ensemble is extrapolated as an ensemble of a particle's stochastic trajectories. In this sense, the average over the esemble trajectories will be its corresponding thermodynamic quantity. The subject was explicitly studied by Seifert for an over-damped Brownian particle in the one-dimensional case. In his work, he defined a first law-like balance between the applied work, the change in internal energy, and the dissipative heat along a stochastic trajectory. Seifert was able to show that the total "entropy like production" (TEP), denoted as $\Delta s_{\text {tot }}$, along a single stochastic trajectory satisfies the integral fluctuation theorem (IFT) which is expressed as $\left\langle e^{-\Delta s_{t o t}}\right\rangle=1$, for any initial condition when the particle is arbitrarily driven by time-dependent external forces over a finite time interval (the transient case). He also showed, in the nonequilibrium steady state over a finite time interval that, a stronger fluctuation theorem, called the detailed fluctuation theorem (DFT) holds, that is, $P\left(\Delta s_{t o t}\right) / P\left(-\Delta s_{\text {tot }}\right)=e^{\Delta s_{\text {tot }}}$; where $P\left(\Delta s_{\text {tot }}\right)$ is the probability of the entropy-like function given along a forward trajectory and $P\left(-\Delta s_{\text {tot }}\right)$ is that given along a backward trajectory. In this context, using the concepts of stochastic thermodynamics and the definition of the entropy-like function along a single stochastic trajectory, Saha et al. Saha et al. (2009) proved that, even in the transient case the DFT for a Brownian particle in a harmonic trap also holds for two exactly solvable models. Finally, we would like to comment that over the last three years, the FTs and the JE have also been explored and verified for a charged Brownian particle in a electromagnetic field Jayannavar \& Sahoo (2007); Jiménez-Aquino et al. (2008; 2009; 2010); Jiménez-Aquino (2010); Saha \& Jayannavar (2008); Roy \& Kumar (2008). In this section we briefly study two transient fluctuation theorems, the work and total entropy-like production, for a Brownian harmonic oscillator in the high friction limiting case, taking also into account the presence of an electromagnetic field. 


\subsection{Transient work-fluctuation theorem}

\subsubsection{Harmonic oscillator's Langevin equation}

Let us consider a charged Brownian particle with mass $m$ in a harmonic trap with a time-dependent position vector $\mathbf{r}^{*}(t)$ of its center, that is $\left.V(\mathbf{r}, t)=(k / 2) \mid \mathbf{r}-\mathbf{r}^{*}\right)\left.\right|^{2}$, with $k$ as a constant. It will be considered that for $t=0$, the potential minimum is located at the origin, $\mathbf{r}_{0}^{*}=0$, whereas for $t>0$, it moves with arbitrary velocity $\mathbf{v}^{*}(t)$. For this Brownian harmonic oscillator, the corresponding Langevin equation in the over-damped approximation reads

$$
\frac{d \mathbf{r}}{d t}=-\gamma \mathbf{r}+\gamma \mathbf{r}^{*}+\alpha^{-1} \boldsymbol{\xi}(t)
$$

where $\gamma=k / \alpha, \alpha$ is the friction coefficient, and $\boldsymbol{\xi}(t)$ represents the fluctuating force produced by the surrounding medium (the bath) at temperature $T$, which satisfies the properties of Gaussian white noise with zero mean value $\left\langle\xi_{i}(t)\right\rangle=0$ and a correlation function given by $\left\langle\xi_{i}(t) \xi_{j}\left(t^{\prime}\right)\right\rangle=2 \lambda \delta_{i j} \delta\left(t-t^{\prime}\right)$, with $i, j=x, y, z$. The parameter $\lambda$ is the noise intensity and acoording to the fluctuation-dissipation theorem it is related to the friction constant by $\lambda=$ $\alpha k_{B} T, k_{B}$ being Boltzmann's constant.

In this work, we adopt the definition of the dimensionless total work done on a system during a finite time interval $\tau$ as given in reference van Zon \& Cohen (2003). In this case

$$
W_{\tau}=\frac{1}{k_{B} T} \int_{0}^{\tau} \mathbf{v}^{*} \cdot \mathbf{F}\left(\mathbf{r}, \mathbf{r}^{*}\right) d t=-\beta k \int_{0}^{\tau} \mathbf{v}^{*} \cdot\left(\mathbf{r}-\mathbf{r}^{*}\right) d t,
$$

where $\beta=k_{B} T$. This definition has been shown to be consistent with the definition of Jarzynski's work in Ref. Jayannavar \& Sahoo (2007); Jiménez-Aquino et al. (2008). To calculate the statistical properties of this total work, we proced further by introducing the change of variable $\mathbf{R}=\mathbf{r}-\langle\mathbf{r}\rangle$, where $\mathbf{R}$ and $\langle\mathbf{r}\rangle$ satisfy the following differential equations van Zon \& Cohen (2003)

$$
\frac{d \mathbf{R}}{d t}=-\gamma \mathbf{R}+\alpha^{-1} \boldsymbol{\xi}(t) \quad \frac{d\langle\mathbf{r}\rangle}{d t}=-\gamma\langle\mathbf{r}\rangle+\gamma \mathbf{r}^{*},
$$

$\langle\mathbf{r}\rangle$ being the deterministic solution of Ec. (37). In terms of the $\mathbf{R}$ variable the total work reads

$$
W_{\tau}=-\beta k \int_{0}^{\tau}\left[\mathbf{v}^{*} \cdot \mathbf{R}+\mathbf{v}^{*} \cdot\left(\langle\mathbf{r}\rangle-\mathbf{r}^{*}\right)\right] d t .
$$

This equation shows that the total work done on the system is a linear function of the stochastic variable R. Accordingly, the statistical properties of this work then correspond to a Gaussian process and therefore its probability distribution can be written as follows

$$
P\left(W_{\tau}\right)=\frac{1}{\sqrt{2 \pi V_{T}(\tau)}} \exp \left(-\frac{\left[W_{\tau}-M_{T}(\tau)\right]^{2}}{2 V_{T}(\tau)}\right),
$$

where we have defined $M_{T}(\tau) \equiv\left\langle W_{\tau}\right\rangle$ as the mean value of the work and $V_{T}(\tau) \equiv$ $\left\langle W_{\tau}^{2}\right\rangle-\left\langle W_{\tau}\right\rangle^{2}$ as its variance. The probability distribution written in Eq. (41) contains the time evolution of the total work from the initial time up to time $\tau$. This fact means that we are studying the distribution corresponding to the transient situation. We will use the subscript $T$ for all quantities in the transient case. Taking into account that $\langle\mathbf{R}\rangle=0$, the work mean value now reads

$$
M_{T}(\tau)=-\beta k \int_{0}^{\tau} \mathbf{v}^{*} \cdot\left(\langle\mathbf{r}\rangle-\mathbf{r}^{*}\right) d t
$$


Following the algebraic steps given by van Zon and Cohen van Zon \& Cohen (2003), it can be shown that the work mean valeu and its corresponding variance are given respectively by

$$
\begin{aligned}
& M_{T}(\tau)=\beta k \int_{0}^{\tau} d t^{\prime} \int_{0}^{t^{\prime}} e^{-\gamma\left(t^{\prime}-t^{\prime \prime}\right)} \mathbf{v}^{*}\left(t^{\prime}\right) \cdot \mathbf{v}^{*}\left(t^{\prime \prime}\right) d t^{\prime \prime} . \\
& V_{T}(\tau)=2 \beta k \int_{0}^{\tau} d t^{\prime} \int_{0}^{t^{\prime}} e^{-\gamma\left(t^{\prime}-t^{\prime \prime}\right)} \mathbf{v}^{*}\left(t^{\prime}\right) \cdot \mathbf{v}^{*}\left(t^{\prime \prime}\right) d t^{\prime \prime},
\end{aligned}
$$

and therefore $V_{T}(\tau)=2 M_{T}(\tau)$. Making use of Eq. (41) we get the final result

$$
\frac{P\left(W_{\tau}\right)}{P\left(-W_{\tau}\right)}=e^{W_{\tau}}
$$

This was precisely the result corroborated experimentally by Wang et al. Wang et al. (2002), before the work of van Zon and Cohen van Zon \& Cohen (2003).

\subsubsection{Harmonic oscillator's Langevin equation in an electromagnetic field}

Recently, the study of van Zon and Cohen, has been extended for an electrically charged Brownian particle in an electromagnetic field Jiménez-Aquino (2010). It is not our purpose in this section to write alegebraic details to obtain the results. We suggest the reader interested in the explicit calculations to read Ref. Jiménez-Aquino (2010). When the above Brownian particle is charged and under the action of constant magnetic field orthogonal to the electric field which lies on the $x-y$ plane, the Langevin equation can be split into two independent processes, one is given on the $x-y$ plane perpendicular to the magnetic field and the other one along the $z$-axis parallel to the magnetic field. Along the $z$-axis, the Langevin equation is the same $z$-component of the Langevin equation studied in Ref. van Zon \& Cohen (2003). We can define all the vectors lying on the plane as $\mathbf{x}=(x, y)$ the position vector, $\mathbf{u}=\left(v_{x}, v_{y}\right)$ the velocity vector, $\boldsymbol{\eta}(t)=\left(\xi_{x}, \xi_{y}\right)$ the fluctuating force, and $\mathbf{E}(t)=\left(E_{x}, E_{y}\right)$ the electric field. The two-dimensional harmonic potential and its corresponding harmonic force are defined as $\mathcal{U}(\mathbf{x}, t)=(k / 2)\left|\mathbf{x}-\mathbf{x}^{*}\right|^{2}$ and $\mathcal{F}\left(\mathbf{x}, \mathbf{x}^{*}\right)=-k\left(\mathbf{x}-\mathbf{x}^{*}\right)$. In this case, the electric field can be interpreted as the responsible to drag the center of the harmonic trap, hence the position vector of the center in the harmonic trap can be written as $\mathbf{x}^{*}=(q / k) \mathbf{E}(t)$. Again for $t=0$, $\mathbf{x}_{0}^{*}=0$, whereas for $t>0, \mathbf{u}^{*}(t) \equiv \dot{\mathbf{x}}^{*}(t)=(q / k) \dot{\mathbf{E}}(t)$ so that. the responsible for dragging of the minimum potential is the electric field rate of change. So, the planar Langevin equation in the over-damped approximation can be written as

$$
\frac{d \mathbf{x}}{d t}=-\Lambda \mathbf{x}+\Lambda \mathbf{x}^{*}+k^{-1} \Lambda \boldsymbol{\eta}(t)
$$

$\Lambda$ is a matrix given by

$$
\Lambda=\left(\begin{array}{cc}
\widetilde{\gamma} & \widetilde{\Omega} \\
-\widetilde{\Omega} & \widetilde{\gamma}
\end{array}\right)
$$

with $\widetilde{\gamma}=k / \alpha\left(1+C^{2}\right), \widetilde{\Omega}=k C / \alpha\left(1+C^{2}\right)$, and $C=q B / c \alpha$ is a dimensionless factor. Now, the total dimensionless work will be in this case

$$
W_{\tau}=-\beta k \int_{0}^{\tau} \mathbf{u}^{*} \cdot\left(\mathbf{x}-\mathbf{x}^{*}\right) d t,
$$


To calculale the statistical properties of this stochastic Gaussian work we again introduce the change of variable $\mathbf{X}=\mathbf{x}-\langle\mathbf{x}\rangle$. Following the steps given in Ref. Jiménez-Aquino (2010), it is also possible to show that the work mean value and its variance are then

$$
\begin{aligned}
& M_{T}(\tau)=\beta k \int_{0}^{\tau} d t^{\prime} \int_{0}^{t^{\prime}} e^{-\widetilde{\gamma}\left(t^{\prime}-t^{\prime \prime}\right)} \mathbf{V}^{*}\left(t^{\prime}\right) \cdot \mathbf{V}^{*}\left(t^{\prime \prime}\right) d t^{\prime \prime}, \\
& V_{T}(\tau)=2 \beta k \int_{0}^{\tau} d t^{\prime} \int_{0}^{t^{\prime}} e^{-\widetilde{\gamma}\left(t^{\prime}-t^{\prime \prime}\right)} \mathbf{V}^{*}\left(t^{\prime}\right) \cdot \mathbf{V}^{*}\left(t^{\prime \prime}\right) d t^{\prime \prime},
\end{aligned}
$$

where $\mathbf{V}^{*}(t) \equiv \mathcal{R}(t) \mathbf{u}^{*}(t)$ and $\mathcal{R}(t)$ is a rotation matrix given by

$$
\mathcal{R}(t)=\left(\begin{array}{cc}
\cos \widetilde{\Omega} t & \sin \widetilde{\Omega} t \\
-\sin \widetilde{\Omega} t & \cos \widetilde{\Omega} t
\end{array}\right),
$$

and thus $V_{T}(\tau)=2 M_{T}(\tau)$. From Eq. (41) we can conclude that the same TFT given by Eq. (45) holds. Therefore, when a system (a charged Brownian particle in a harmonic trap) in the presence of a uniform magnetic field is driven out of equilibrium by an time-varying electric field, the transient work-fluctuation theorem (TFT) also holds.

\subsection{Entropy production fluctuation theorem}

Using the concepts of stochastic thermodynamics established by Seifert Seifert $(2005 ; 2008)$, Saha and collegues Saha et al. (2009) showed in 2009 that even in the transient case the DFT for a Brownian particle in a harmonic trap also holds for two exactly solvable models. These models were studied in the over-damped approximation of the Langevin Dynamics in the one variable case. Last year, Saha's et al. work could be extended to the case of a charged harmonic oscillator in an electromagnetic field by Jiménez-Aquino Jiménez-Aquino (2010). In this paper, also two physical situations for arbitrary time-dependent electric field driven over a finite time interval were also studied, namely: (i) the charged Brownian particle in a two-dimensional harmonic trap subjected to the action of an arbitrary time-dependent electric field and, (ii) the minimum of the harmonic trap arbitrarily dragged by the electric field. We brefly introduce a short comment of stochastic thermodynamics before establishing the entropy-production fluctuation theorem. According to stochastic thermodynamics Seifert (2005; 2008); Sekimoto (1998), the first-law-like balance between the applied work W, the change in internal energy $\Delta U$, and the dissipated heat $Q$ to the bath, can be calculated along a trajectory $\mathbf{x}(t)$ over a finite time interval $t$. This first-law-like reads as $Q=W-\Delta U$, where the work (not dimensionless work) can be calculated from the relation Jarzynski (1997a); Jayannavar \& Sahoo (2007); Saha et al. (2009)

$$
W=\int_{0}^{t} \frac{\partial U\left(\mathbf{x}, t^{\prime}\right)}{\partial t^{\prime}} d t^{\prime}
$$

where $\mathbf{x}$ is the $n$-dimensional position vector. On the other hand, the change in the medium's entropy $\Delta s_{m}$ over the time interval is $\Delta s_{m}=Q / T$ and the nonequilibrium Gibbs entropy-like $S$ of the system in the present problem is defined as Seifert (2005)

$$
S(t)=-\int f(\mathbf{x}, t) \ln f(\mathbf{x}, t) d \mathbf{x}=\langle s(t)\rangle .
$$

This definition suggests the definition of a trajectory-dependent entropy-like function for the particle as $s(t)=-\ln f[\mathbf{x}(t), t]$, where the probability density $f(\mathbf{x}, t)$ is obtained through the 
solution of the associated Fokker-Planck equation and, it is evaluated along the stochastic trajectory. For a given trajectory $\mathbf{x}(t)$, this entropy-like $s(t)$ depends on the given initial data $f\left(\mathbf{x}_{0}\right) \equiv f\left(\mathbf{x}_{0}, 0\right)$, where $f\left(\mathbf{x}_{0}\right)$ is the probability density of the particle at initial time $t=0$, and thus contains information about the whole ensemble. The change in the system's entropy for any trajectory of duration $t$ is $\Delta s=-\ln \left[f(\mathbf{x}, t) / f\left(\mathbf{x}_{0}\right)\right]$. Now, the change in the total entropy-like production along a trajectory over a finite time interval $t$ is shown to be Seifert $(2005 ; 2008) \Delta s_{t o t}=\Delta s_{m}+\Delta s$. Using this definition, Seifert derived the integrated fluctuation theorem, $\left\langle e^{-\Delta s_{t o t}}\right\rangle=1$, where the angular brackets denote average over the statistical ensemble of realizations, or over the ensemble of finite time trajectories Seifert (2005; 2008). Also he showed that in the nonequilibrium steady state over a finite time interval, the DFT holds. The latter is stated as

$$
\frac{P\left(\Delta s_{t o t}\right)}{P\left(-\Delta s_{t o t}\right)}=e^{\Delta s_{t o t}}
$$

This theorem has also been proved, even in the transient case, for a Brownian particle in a harmonic trap only if the system is initially prepared in equilibrium Saha et al. (2009). This was also the case for a charged harmonic oscillator in an electromagnetic field as studied by Jiménez-Aquino (2010). In both papaers Jiménez-Aquino (2010); Saha et al. (2009), the proof of the DFT has been given for two general physical situations, when the system is initially distributed with a canonical distribution at equilibrium with the thermal bath. The relation $\sigma_{s}^{2}=2\left\langle\Delta s_{\text {tot }}\right\rangle$ is shown to be valid, being the main requirement for the validity of the DFT. However, as it has beeen pointed out by Saha et al. Saha et al. (2009), when the probability density $P\left(\Delta s_{t o t}\right)$ is not Gaussian the DFT for the total entropy-like producton does not hold. To this respect, it is important to take into account the recent commets of Shargel Shargel (2010) about Saha's contribution, namely: as proved by Saha et al., the transient fluctuation theorem (TFT), given by Eq. (54) for a Brownian particle in a harmonic potential and driven by an arbitrary time-dependent force, is only valid if the particle is initially in thermal equilibrium. However, this is a surprising result because according to Shargel, Eq. (54) fails to distinguish between both the forward and backward path measures and the forward and backward entropy-like production, each of which are different due to the time-dependent driving. Certainly, in those works Jiménez-Aquino (2010); Saha et al. (2009), the TFT holds in a very particular case that strongly depends of the form on which the initial distribution has been constructed. The latter is constructed on the basis of a harmonic potential, but any other potential or initial distribution the TFT breaks down.

Respect to the stochastic thermodynamics concepts briefly outlined above, we must mention a critical analysis given very recently in Velasco et al. (2011): as addressed by the authors, the usual concepts of thermodynamics when extrapolated to the study of thermodynamic of small systems labelled under the strange name of "stochastic thermodynamics", some care should be taken into account when concepts of entropy and related quantities out of equilibrium are used in such a description. The authors further suggest to review the books on the thermodynamics of small systems formulated over fifty years ago by T. L. Hill Hill $(1963 ; 1964 ; 1968)$. Hill's theory is formulated upon a precise and conventional theory of themodynamics of small systems, and based on the fundamental ideas of statistical mechanics. To describe non-equilibrium systems consistent with thermodynamics, the theory relies upon local equilibrium states, so that the calculation of the entropy production in irreversible processes must be the same as those available for thermodynamic systems. In this context, it seems to be the case that the connection of Hill's theory with systems described by 
averaging procedure, like Jarzynski equality, has been given first by J. Carrete and collegues Carrete et al. (2008) in 2008. This pioneer work opens other possibilities to explore the FTs described by the so-called "stochastic thermodynamics" theory.

\section{References}

Aad, G et al. (2010). Observation of a Centrality-Dependent Dijet Asymmetry in Lead-Lead Collisions at $\sqrt{s_{N N}}=2.76 \mathrm{TeV}$ with ATLAS Detector at the LHC, Phys. Rev. Lett. Vol. 105: 252303.

Aamodt, K. et al. (2010). Elliptic Flow of Charged Particles in Pb-Pb Collisions at $\sqrt{s_{N N}}=$ 2.76, Physical Review Letters Vol. 105: 252302.

Agarwal, R. K., Yun, K. Y. \& Balakrishnan, R. (2001). Beyond Navier-Stokes: Burnett equations for flows in the continuum-transition regime, Physics of Fluids Vol. 13: 3061-3085.

Alfven, H \& Falthammar, C. G. (1963). Cosmical Electrodynamics Fundamental Principles, Oxford University Press.

Alsmeyer H. (1976). Density profiles in Argon and Nitrogen shock waves measured by the absorption of an electron beam, Journal of Fluid Mechanics Vol. 74: 497-513.

Arnold, V. I, (1998) Topological Methods in Hydrodynamics, Springer.

Balbus, S. A. \& Hawley, J. F. (1998), Instability, turbulence, and enhaced transport in accretion disks, Reviews of Modern Physics Vol. 70: 1-53.

Balescu, R. (1988). Transport Processes in Plasmas: Vol. 1 Classical Transport, North Holland.

Bhatnagar, P. L., Gross, E. P., Krook, M. (1954). A model for collision processes in gases. I: Small amplitude processes in charged and neutral one-component systems, Physical Review Vol. 94: 511-525.

Bird, G. A. (1994). Molecular Gas Dynamics and the Direct Simulation of Gas Flows, Oxford-Clarendon.

Bjorken, J. D. (1983). Highly relativistic nucleus-nucleus collisions: The central rapidity region, Physical Review D Vol. 27: 140-151.

Blickle, V., Speck, T., Helden, L., Seifert, U. \& Bechinger, C. (2006). Thermodynamics of a Colloidal Particle in a Time-Dependent Nonharmonic Potential, Physical Review Letters Vol. 96: 070603.

Bobylev, A. V. (2008). Generalized Burnett hydrodynamics, Journal of Statistical Physics Vol. 132: $569-580$.

Bobylev, A. V., Bisi, Cassinari, M. P. \& Spiga, G. (2001). Shock wave structure for generalized Burnett equations, Physics of Fluids Vol. 23: 030607.

Bobylev, A. V. (2006). Instabilities in the Chapman-Enskog expansion and hyperbolic Burnett equations, Journal of Statistical Physics Vol. 124: 371-399.

Braginskii, S. I. (1965) Transport Processes in a Plasma, in Leontouech, M. A. (ed.), Reviews of Plasma Physics Vol. 1, Consultants Bureau, New York, p. 45.

Brenner, H. (2005). Navier-Stokes revisited, Physica A Vol. 349: 60-132.

Brenner, H. (2006). Fluid mechanics revisited, Physica A Vol. 370: 190-224.

Brenner, H. (2010). Bi-velocity transport processes. Single-component liquid and gaseous continua, Physica A Vol. 389: 1297-1316.

Brenner, M. P., Hilgenfeldt, S. \& Lohse, D. (2002). Single-bubble sonoluminescence, Review of Modern Physics Vol. 72: 425-484.

Brey, J, J., Dufty, J. W., Kim, C. S., \& Santos, A. (1998). Hydrodynamics for granular flow at low density, Physical Review E Vol. 58: 4638-4653. 
Burnett, D. (1935). The distribution of velocities in a slightly non-uniform gas, Proccedings of the London Mathematical Society Vol. 39: 385-430.

Bustamante, C., Liphardt, J. \& Ritort, F. (2005). The Nonequilibrium Thermodynamics of Small Systems, Physics Today Vol. 58: 43-48.

Campisi, M., Talkner, P. \& Hänggi, P. (2009). Fluctuation Theorem for Arbitrary Open Quantum Systems, Physical Review Letters Vol. 102: 210401.

Cao, Elliott, E., Joseph, J., Wu, H., Petricka, J., Schäfer, T. \& Thomas, J. E. (2011). Universal Quantum Viscosity in a Unitary Fermi Gas, Science Vol. 331: 58.

Carberry, D. M., Reid, J. C., Wang, G. M., Sevick, E. M., Searles, D. J. \& Evans, D. J. (2004). Fluctuations and Irreversibility: An Experimental Demonstration of a Second-Law-Like Theorem Using a Colloidal Particle Held in an Optical Trap, Physical Review Letters Vol. 92: 140601.

Carrete, J., Varela, L. M. \& and Gallego, L. J. (2008). Nonequilibrium nanothermodynamics, Physical Review E Vol. 77: 022102.

Cercignani, C. (1988). The Boltzmann Equation and its Applications, Springer-Verlag.

Cercignani, C., Illner, R. \& Pulvirenti, M. (1994). The Mathematical Theory of Dilute Gases, Springer-Verlag.

Chandrasekhar, S. (1960). Plasma Physics, University of Chicago Press.

Chapman, S. \& Cowling, T. G. (1970). The Mathematical Theory of Non-Uniform Gases, Cambridge University Press.

Collin, D., Ritort, F., Jarzynski, C., Smith, S. B., Tinoco Jr, I., \& Bustamante, C. (2005). Verification of the Crooks fluctuation theorem and recovery of RNA folding free energies, Nature Vol. 437: 231-234.

Cowling, T. G. (1957). Magnetohydrodynamics, Interscience Publ. Co..

Crooks, G. E. (2000). Path-ensemble averages in systems driven far from equilibrium, Physical Review E Vol. 61: 2361-2366.

Crooks, G. E. (1999). Entropy production fluctuation theorem and the nonequilibrium work relation for free energy differences, Physical Review E Vol. 60: 2721-2726.

Currie, L. G. (1974). Fundamental Mechanics of Fluids, Mc-Graw-Hill.

Erwin, D. A., G.C. Pham-Van-Diep, G. C. \& E.P. Muntz, E. P. (1991). Nonequilibrium gas flows: A detailed validation of Monte Carlo direct simulation for monatomic gases, Physics of Fluids A Vol. 3: 697-705.

Esposito, M., Harbola, U. \& Mukamel, S. (2010) Nonequilibrium fluctuations, fluctuation theorems, and counting statistics in quantum systems, Reviews of Modern Physics Vol. 81: 1665-1698.

Esposito, M. \& Van den Broeck, C. (2010). Three Detailed Fluctuation Theorems, Physical Review Letters Vol. 104: 090601.

Evans, D. J. \& Searles, D. J. (2002). The fluctuation theorem, Advances in Physics, Vol. 51: 1529-1585.

Evans, D. J. \& Searles, D. J. (1994). Equilibrium microstates which generate second law violating steady states, Physical Review E Vol. 50: 1645-1648.

Evans, D. J., Cohen, E. G. D. \& Morris, G. P. (1993). Probability of second law violations in shearing steady states. Physical Review Letters Vol. 71: 2401-2404.

Eu, B. C. (1998). Nonequilibrium Statistical Methods, Kluwer.

Feshbach, H. \& and F. Villars, F. (1958). Elementary Relativistic Wave Mechanics of Spin 0 and Spin 1/2 Particles, Reviews of Modern Physics Vol. 30: 24-45. 
Ferziger, J. H. \& Kaper, H. P. (1972). Mathematical Theory of Transport Processes in Gases North-Holland.

Feynman, R. P. (1955). Applications of Quantum Mechanics to Liquid Helium, in Gorter, C. J. (ed.), Progress in Low Temperature Physics, vol. I, North Holland, Amsterdam, chapter II.

Fefferman, C. L. (2000). Existence \& Smoothness of the Navier-Stokes Equations. Manuscript available at:http://www.claymath.org/millennium/.

Fickett, W, \& Davis, W. C. (2000). Detonation Theory and Experiment, Dover.

Fiscko, K. A. \& D.R. Chapman, D. R. (1989). Comparison of Burnett, Super-Burnett and Monte Carlo solutions for hypersonic shock structure, in Muntz, E. P., Weaver, D. P. \& Campbell, D. H. (eds.), Rarefied Gas Dynamics, vol. 18, AIAA, Washington D. C., pp. 374-395.

Foch Jr., J. D. (1973). On higher order hydrodynamic theories of shock structure, Acta Physica Austriaca suppl. X: 123-140.

Foch Jr., J. D. \& Simon, C. E. (1977). Numerical integration of the Burnett equations for shock structure in a Maxwell gas, in J. L. Potter (ed.), Rarefied Gas Dynamics, Progress in Astronautics and Aeronautics, vol. 51, AIAA, New York, pp. 493-500.

Fomin, N. A. (2010). 110 Years of Experiments on Shock Tubes, Journal of Engineering and Thermophysics Vol. 83: 1118-1135.

Gallavotti, G. \& Cohen, E. G. D. (1995). Dynamical Ensembles in Nonequilibrium Statistical Mechanics, Physical Review Letters Vol. 74: 2694-2697.

García-Colín \& Dagdug, L. (2009). The Kinetic Theory of Inert Dilute Plasmas, Springer-Verlag.

García-Colín, L. S., Velasco, R. M., \& Uribe, F. J. (2008). Beyond the Navier-Stokes equations: Burnett hydrodynamics, Physics Reports Vol. 465: 149-189.

García-Colín, L. S., Velasco, R. M. \& Uribe, F. J. (2004). Inconsistency in the Moment's method for solving the Boltzmann equation, Journal of Non-Equilibrium Thermodynamics Vol. 29: 255-277.

Garnier, E. \& Ciliberto, S. (2005). Nonequilibrium fluctuations in a resistor, Physical Review E Vol. 71: 060101.

Gilbarg, D. \& Paolucci, D. (1953). The structure of shock waves in the continuum theory of fluids, J. Ration. Mech. Anal. Vol. 2 617-642.

Gilbarg, D. (1951). The Existence and Limit Behavior of the One-Dimensional Shock Layer, American Journal of Mathematics Vol. 73: 256-274.

Gnoffo, P. A. (1999). Planetary-entry gas dynamics, Annual Review of Fluid Mechanics Vol. 31: 459-494.

Goncharov, V. N., Gotchev, O. V., Vianello, E., Bohely, T. R., Knauer, J. P., McKenty, P. W., Radha, P. B., Regan, S. P., Sangster, T. C., S. Skupsky, S., Smalyuk, V. A., Betti, R., McCrory, R. L., Meyerhofer, D. D. \& C. Cherfils-Clérouin, C. (2006). Early stage of implosion in inertial confinement fusion: Shock timing and perturbation evolution, Physics of Plasmas Vol. 13: 012702.

Grad, H. (1958). Principles of the kinetic theory of gases, in Flügge, D. (ed.), Handbuch der Physik, Springer-Verlag, Berlin, pp. 205-294.

Greenshields, C. J. \& and J. M. Reese, J. M. (2007). The structure of shock waves as a test of Brenner's modications to the Navier-Stokes equations, Journal of Fluid Mechanics Vol. 580: 407-429. 
Helbing, D. (2001). Traffic and related self-driven many-particle systems, Reviews of Modern Physics Vol. 73: 1068-1141.

Hill, T. L. (1963). Thermodynamics of small sytems, Part I, Benjamin.

Hill, T. L. (1964). Thermodynamics of small sytems, Part II Benjamin.

Hill, T. L. (1968). Thermodynamics for Chemist and Biologist, Addison Wesley.

Holian, B. L., Mareschal, M. \& Ravelo, R. (2011). Burnett-Cattaneo continuum theory for shock waves, Physical Review E Vol. 83: 026703.

Holian, B. L. \& Lomdahl, P. S. (1998). Plasticity Induced by Shock Waves in Nonequilibrium Molecular-Dynamics Simulations, Science Vol. 280: 2085-2088.

Holian, B. L., Patterson, C. W., Mareschal, M. \& Salomons, E. (1993). Modeling shock waves in an ideal gas: Going beyond the Navier-Stokes level, Physical Review E Vol. 47: R24-R27.

Holian B. L., Hoover, W. G., Moran B. \& Straub B. K. (1980). Shock-wave structure via non-equilibrium molecular-dynamics and Navier-Stokes continuum mechanics, Physical Review A Vol. 22: 2798-2808.

Holmes, P., Lumley, J. L. \& Berkooz, G. (1998). Turbulence, Coherent Structures, Dynamical Systems and Symmetry, Cambridge University Press.

Hoover, Wm. G. \& Hoover, C. G. (2010). Shock waves and Local Hydrodynamics; Failure of the Navier-Stokes Equations, Macias, A. \& Dagdug, L. (eds.), in New trends in Statistical Physics: Festschrift in Honor of Leopoldo García-Colín 80 ${ }^{\text {th }}$ Birthday, World Scientific, Singapore, pp. 15-26.

Hoover, Wm. G. \& Hoover, C. G. (2010). Well-posed two-temperature constitutive equations for stable dense fluid shock waves using molecular dynamics and generalizations of Navier-Stokes-Fourier continuum mechanics, Physical Review E Vol. 81: 046302.

Hoover, Wm. G. (2006). Smooth Particle Applied Mechanics: The State of the Art., World Scientific.

Horikoshi, M., Nakajima, S., Ueda, M. \& Mukuyama, T. (2010). Measurement of Universal Thermodynamic Functions for a Unitary Fermi Gas, Science Vol. 327: 442-445.

Huang, K. (1964). in de Boer, J. \& Uhlenbeck, G. E. (eds.), Studies in Statistical Mechanics, vol. II, North Holland, Amsterdam, pp. 2-106.

Hwa, R. C. (1974). Statistical description of hadron constituents as a basis for the fluid model of high-energy collisions, Physical Review D Vol. 10: 2260-2268.

Irving, J. H. \& Kirkwood, J. G. (1950). The Statistical Mechanical Theory of Transport Processes. IV. The Equations of Hydrodynamics, Journal of Chemical Physics Vol. 18: 817-829.

Irving, J. H. \& Zwanzig, R. W. (1951). The Statistical Mechanical Theory of Transport Coefficients. 6. Quantum Hydrodynamics, Journal of Chemical Physics, Vol. 19: 1173-1180.

Jackson, J. D. (1962). Classical Electrodynamics, John Wiley \& Sons.

Jarzynski, C. (1997) Nonequilibrium Equality for Free Energy Differences, Physical Review Letters Vol. 78: 2690-2693.

Jarzynski, C. (1997) Equilibrium free-energy differences from nonequilibrium measurements: A master-equation approach, Physical Review E Vol 56: 5018-5035.

Jarzynski, C. (2007). Lect. Notes Phys. Vol. 711 201-216.

Jayannavar, A. M. \& Sahoo, M. (2007). Charged particle in a magnetic field: Jarzynski equality, Physical Review E Vol. 75: 032102. 
Jiménez-Aquino, J. I., Velasco, R. M. \& Uribe, F. J. (2008). Dragging of an electrically charged particle in a magnetic field, Physical Review E Vol. 78: 032102.

Jiménez-Aquino, J. I., Velasco, R. M. \& Uribe, F.J. (2009). Fluctuation relations for a classical harmonic oscillator in an electromagnetic field, Physical Review E Vol. 79: 061109.

Jiménez-Aquino, J. I, Uribe, F. J., \& Velasco, R. M. (2010). Work-fluctuation theorems for a particle in an electromagnetic field, J. Phys. A: Math. Theor. Vol. 43: 255001.

Jiménez-Aquino, J. I. (2010). Entropy production theorem for a charged particle in an electromagnetic field, Physical Review E Vol. 82: 051118.

Jin, S. \& Slemrod, M. (2001). Regularization of the Burnett equations for rapid granular flows via relaxation, Physica D-nonlinear Phenomena Vol. 150: 207-218.

Jin, S. \& Slemrod, M. (2001). Regularization of the Burnett equations via relaxation, Journal of Statistical Physics Vol. 103: 1009-1033.

Saha, A. \& Jaynnavar, A. M. (2008). Nonequilibrium work distributions for a trapped Brownian particle in a time-dependent magnetic field, Physical Review E Vol. 77: 022105.

Kadanoff, L. P. \& Baym, G. (1962). Quantum Statistical Mechanics, Benjamin.

Kauzmann, W. (1966). Kinetic Theory of Gases, Benjamin. Khalatnikov, I. M. (1965). Introduction to the Theory of Superfluidity, Benjamin.

Kovtun, P., Son, D. T. \& Sterinets, A. O. (2005). Physical Review Letters Vol. 94: 11160.

Khalatnikov, I. M. (1965). Introduction to the Theory of Superfluidity, Benjamin.

Kulsrud, R. M. (2005). Plasma Physics for Astrophysics, Princeton University Press.

Landau, Izv. Akad. Nauk. USSR 17, 51 (1953).

Landau, L. D. (1941). The Theory of Superfluidity of Helium II, J. Phys. USSR Vol. 5: 71-90.

Landau, L. D.(1986). Fluid Mechanics, Pergamon Press.

Lechner, W., Oberhofer, H., Dellago, C. \& Geissier, P. L. (2006). Equilibrium free energies from fast-switching trajectories with large time steps, Journal of Chemical Physics Vol. 124: 044113.

London, F. (1964). Superfluids Vol. II, Macrocopic Theory of Superfluid Helium, Dover.

Luo, L. \& Thomas, J. E. (2009). Thermodynamic Measurements in a Strongly Interacting Fermi Gas, Journal of Low Temperature Physics Vol. 154: 1-29.

Liphardt, J., Dumont, S., Smith, S. B., Tinoco Jr., I. \& Bustamente, C. (2002). Equilibrium Information from Nonequilibrium Measurements in an Experimental Test of Jarzynski's Equality, Science Vol. 296: 1832-1835.

Madelung E. (1927) Quantentheorie in hydrodynamischer form, Zeits. für Physik Vol. 40, $322-$.

McCourt, F. R. W., Beenakker, J. J. M., Köhler, W. E. \& Kuščer, I. (1990). Nonequilibrium Phenomena in Polyatomic Gases, Clarendon Press.

Moratto, V. \& García-Colín, L. S. (2011), Relaxation time for the Temperature in a dilute binary mixture from classical kinetic theory, J. Non-equilibrium Thermodynamics (in the press).

Mori, H., Oppenheim, I. \& Ross, J. (1962). Wigner Functions and Transport Theory, in G. E. Uhlenbeck \& J. de Boer (eds.), Studies in Statistical Mechanics, North Holland, Amsterdam.

Mott-Smith, H. M. (1951). The solution of the Boltzmann equation for a shock wave, Physical Review Vol. 82: 885-892.

Müller, I. \& T. Ruggeri, T. (1993). Extended Thermodynamics, Springer.

Onsager, L., Unpublishd Remark at Low Temperature Conference at Shelter Island (1948). See Putterman (1974). 
Pham-Van-Diep, C. G., Erwin, D. A. \& Muntz, E. P. (1991). Testing continuum descriptions of low-Mach-number shock structures, Journal of Fluid Mechanics Vol. 232: 403-413.

Piran, T. (2004). The physics of gamma-ray bursts, Reviews of Modern Physcis Vol. 76: 1144-1210.

Procacci, P., Marsili, S., Barducci, A., Signorini, G. F. \& Chelli, R. (2006). Crooks equation for steered molecular dynamics using a Nosé-Hoover thermostat, Journal of Chemical Physics Vol. 125: 164101.

Putterman, S. J. (1974). Superfluid Hydrodynamics, North Holland.

Richmyer, R. D. (1978). Principles of Advanced Mathematical Physics, Vol1, Springer-Verlag.

Reif, F. (1966). Fundamentals of Thermal and Statistical Physics, Mc Graw Hill.

Résibois, P. \& Leener, M. D. (1977). Classical Kinetic Theory of Fluids, Wiley.

Ritort, F. (2009). Fluctuation in open systems, Physics Vol. 2: 43.

Rosenau, P. (1989). Extending hydrodynamics via the regularizations of the Chapman-Enskog expansion, Physical Review A Vol. 40: 7193âĂŞ7196.

Roy, D. \& Kumar, N. (2008). Langevin dynamics in crossed magnetic and electric fields: Hall and diamagnetic fluctuations, Physical Review E Vol. 78: 052102.

Saito, K. \& Dhar, A. (2007). Fluctuation Theorem in Quantum Heat Conduction, Physical Review Letters Vol. 99: 180601.

Saha, A., Lahiri, S. \& Jayannavar, A. M. (2009). Entropy production theorems and some consequences, Physical Review E Vol. 80: 011117.

Salomons, E., \& Mareschal, M. (1992). Usefulness of the Burnett description of strong shock-waves, Physical Review Letters Vol. 69: 269-272.

Searles, D. J. \& Evans, D. J. (2000). Ensemble dependence of the transient fluctuation theorem, Journal of Chemical Physics Vol. 113: 3503-3509.

Seifert, U. (2005). Entropy Production along a Stochastic Trajectory and an Integral Fluctuation Theorem, Physical Review Letters Vol. 95: 040602.

Seifert, U. (2008). Stochastic thermodynamics: principles and perspectives, Eur. Phys. J. B Vol. 64: 423-431.

Sekimoto, K. (1988). Langevin Equation and Thermodynamics, Prog. Theor. Phys. Suppl. 130: 17-27.

Sela, N \& Goldhirsch, I. (1998). Hydrodynamic equations for rapid flows of smooth inelastic spheres, to Burnett order, Journal of Fluid Mechanics Vol. 361: 41-74.

Sevick, E. M., Prabhakar, R., Williams, S. R. \& Searles, D. J. (2008). Fluctuation Theorems, Annu. Rev. Phys. Chem. Vol. 59: 603-633.

Shargel, B. H. (2010). The measure-theoretic identity underlying transient fluctuation theorems, J. Phys. A: Math. Theor. Vol. 43: 135002.

Schäfer, T. \& Teaney, D. (2009). Nearly perfect fluidity: from cold atomic gases to hot quak-gluon plasmas. Rep. Prog. Phys. Vol. 72, 126001.

Slemrod, M. (1998). Renormalization of the Chapman-Enskog expansion: Isothermal fluid flow and Rosenau saturation, Journal of Statistical Physics Vol. 91: 285-305.

Sone, Y. (2002). Kinetic Theory and Fluid Dynamics, Birkhäuser.

Spitzer, L. (1956). The Physics of Fully Ionized Gases, Interscience Wiley.

Struchtrup, H. (2005). Macroscopic Transport Equations for Rarefied Gas Flows, Springer-Verlag.

Tietz, C., Schuler, S., Speck, T., Seifert, U. \& Wrachtrup, J. (2006). Thermodynamics of a Colloidal Particle in a Time-Dependent Nonharmonic Potential, Physical Review Letters Vol. 97: 050602. 
Trepagnier, E. H., Jarzynski, C., Ritort, F., Crooks, G. E., Bustamante, C. J. \& Liphardt, J. (2004). Experimental Test of Hatano and Sasa's Nonequilibrium Steady-State Equality, Proc. Natl. Acad. Sciences Vol. 101: 15038-15041.

Truesdell C. \& Müncaster, R. G. (1980). Fundamentals of Maxwell's Kinetic Theory of a Simple Monatomic Gas, Academic Press.

Uribe, F. J., Hoover, Wm. G. \& Hoover, C. G. (2011). Maxwell and Cattaneo's Time-Delay Ideas Applied to Shockwaves and the Rayleigh-Bénard Problem, arXiv:1102.2560v2 [physics.flu-dyn].

Uribe, F. J. (2011). The Shock Wave Problem Revisited: The Navier-Stokes Equations and Brenner's Two Velocity Hydrodynamics, in Gorban, A. N. \& Roose, D. (eds.), Coping with Complexity: Model Reduction and Data Analysis, Springer, Berlin, pp. 207-229. In eqs. (11) and (14) the changes $T \rightarrow T(u)$ and $u(T) \rightarrow u$ should be made.

Uribe, F. J. (2001). Understanding dilute gases: Going beyond the Navier-Stokes equations, in Ramos, E., Cisneros, G., Fernández-Flores, R., Santillán-González A. (eds.), Computational Fluid Mechanics, World Scientific, Singapore, pp. 255-264.

Uribe, F. J., Velasco, R. M., García-Colín, L. S. \& Díaz-Herrera, E. (2000). Shock wave profiles in the Burnett approximation, Physical Review E Vol.62: 6648-6666.

Uribe, F. J., Velasco, R. M. \& García-Colín, L. S. (1998). Burnett description of strong shock waves, Physical Review Letters Vol. 81: 2044-2047.

Van Kampen, N. G. \& Felderhof, B. U. (1967). Theoretical Methods in Plasma Physics, North Holland.

van Zon, R. \& and Cohen, E. G. D. (2004). Extended heat-fluctuation theorems for a system with deterministic and stochastic forces, Physical Review E Vol. 69: 056121.

van Zon, R. \& and Cohen, E. G. D. (2003). Stationary and transient work-fluctuation theorems for a dragged Brownian particle, Physical Review E Vol 67: 046102.

Velasco, R. M., García-Colín, L. S. \& Uribe, F. J. (2011). Entropy Production: Its Role in Non-Equilibrium Thermodynamics, Entropy Vol. 13: 82-116.

Velasco, R. M., Uribe, F. J. \& García-Colín, L. S. (2002). Inconsistencies in moment methods Physical Review E Vol. 66: 032103.

Villani, C. (2006). A Review of Mathematical Topics in Collisional Kinetic Theory, in S. Friedlander, S. \& D. Serre, D. (eds.), Handbook of Mathematical Fluid Dynamics, vol. 1, Elsevier Science, pp. 71-261.

von Neumann, J. (1955). Mathematical Foundations of Quantum Mechanics, Princeton University Press.

Wang, G. M., Sevick, E. M., Mittag, E., Searles, D. J. \& Evans, D. J. (2002). Experimental Demonstration of Violations of the Second Law of Thermodynamics for Small Systems and Short Time Scales, Physical Review Letters Vol. 89: 050601.

Wigner, E. P. (1932). On the quantum correction for thermodynamic equilibrium, Physical Review Vol. 40: 749-759.

Wilks, J. (1966). The Properties of Liquid and Solid Helium, Oxford University Press, Clarendon.

Wong, C. Y. (2008). Landau hydrodynamics reexamined, Physical Review C Vol. 78: 054902.

Wong, C. Y. (2010). Foundation of Hydrodynamics for Systems with Strong Interactions, in Dagdug, L. et al. (eds.), AIP Conference Proceedings 1312, IV Mexican Meeting in Mathematical and Experimental Physics: Relativistic and Biological Physics, AIP, USA, pp. $39-48$.

Wong, C. Y. (2008). Lectures on Landau Hydrodynamics. arXiv: 0809.0517v1 [nucl-th]. 
Wong, C. Y. (2010). Klein-Gordon in hydrodynamical form, Journal of Mathematical Physics, Vol. 51: 122304.

Woosley, S. E., Heger, A., \& Weaver, T. A. (2002). The evolution and explosion of massive stars, Reviews of Modern Physics Vol. 74: 1016-1071.

Xu, K. \& Josyula, E. (2005). Multiple translational temperature model and its shock structure solution, Physical Review E Vol. 71: 056308.ôRř̌č

Xu, K. \& Li, Z. H. (2004). Microchannel flow in the slip regime: gas-kinetic BGK-Burnett solutions, Journal of Fluid Mechanics Vol. 153: 87-110.

Yarnell, J. L., Arnold, G. P., Bendt, P. J. \& and Kerr, E. C. (1959). Exitations in Liquid Helium-Neutron Scattering Measurements, Physical Review Vol. 113: 1379-1386.

Zel'dovich, Ya. B. \& Raizer, Yu. P. (2002). Physics of Shock Waves and High-Temperature Hydrodynamic Phenomena, Dover.

Zuckerman, D. M. \& Wolf, T. B. (2002). Theory of a Systematic Computational Error in Free Energy Differences, Physical Review Letters Vol. 89: 180602. 


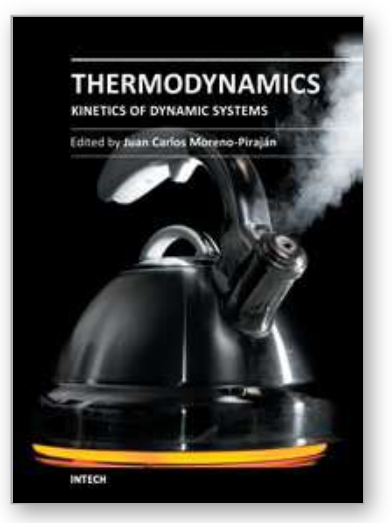

\author{
Thermodynamics - Kinetics of Dynamic Systems \\ Edited by Dr. Juan Carlos Moreno Pirajễin
}

ISBN 978-953-307-627-0

Hard cover, 402 pages

Publisher InTech

Published online 22, September, 2011

Published in print edition September, 2011

Thermodynamics is one of the most exciting branches of physical chemistry which has greatly contributed to the modern science. Being concentrated on a wide range of applications of thermodynamics, this book gathers a series of contributions by the finest scientists in the world, gathered in an orderly manner. It can be used in post-graduate courses for students and as a reference book, as it is written in a language pleasing to the reader. It can also serve as a reference material for researchers to whom the thermodynamics is one of the area of interest.

\title{
How to reference
}

In order to correctly reference this scholarly work, feel free to copy and paste the following:

L. S. Garcia-Colín, J. I. Jiménez-Aquino and F. J. Uribe (2011). Thermohydrodynamics: Where Do We Stand?, Thermodynamics - Kinetics of Dynamic Systems, Dr. Juan Carlos Moreno PirajÃ $i n(E d)$, ISBN: 978953-307-627-0, InTech, Available from: http://www.intechopen.com/books/thermodynamics-kinetics-ofdynamic-systems/thermohydrodynamics-where-do-we-stand-

\section{INTECH}

open science | open minds

\section{InTech Europe}

University Campus STeP Ri

Slavka Krautzeka 83/A

51000 Rijeka, Croatia

Phone: +385 (51) 770447

Fax: +385 (51) 686166

www.intechopen.com

\section{InTech China}

Unit 405, Office Block, Hotel Equatorial Shanghai

No.65, Yan An Road (West), Shanghai, 200040, China

中国上海市延安西路65号上海国际贵都大饭店办公楼405单元

Phone: +86-21-62489820

Fax: $+86-21-62489821$ 
(C) 2011 The Author(s). Licensee IntechOpen. This chapter is distributed under the terms of the Creative Commons Attribution-NonCommercialShareAlike-3.0 License, which permits use, distribution and reproduction for non-commercial purposes, provided the original is properly cited and derivative works building on this content are distributed under the same license. 